\title{
Robust Shortest Path Problems with Two Uncertain Multiplicative Cost Coefficients
}

\section{Changhyun Kwon}

Department of Industrial and Systems Engineering

University at Buffalo, SUNY, USA

\section{Taehan Lee}

Department of Industrial and Information Systems Engineering

Chonbuk National University, Korea

Corresponding author. E-mail: myth0789@jbnu.ac.kr

\section{Paul Berglund}

Norfolk Southern Corporation

Atalanta, Georgia, USA

April 24, 2013

\begin{abstract}
We consider a robust shortest path problem when the cost coefficient is the product of two uncertain factors. We first show that the robust problem can be solved in polynomial time by a dual variable enumeration with shortest path problems as subproblems. We also propose a path enumeration approach using a $K$-shortest paths finding algorithm that may be efficient in many real cases. An application in hazardous materials transportation is discussed and the solution methods are illustrated by numerical examples.
\end{abstract}

Keywords: robust shortest path; budgeted uncertainty; hazardous materials transportation 


\section{Introduction}

For a directed and weighted graph $G(\mathcal{N}, \mathcal{A})$ we are interested in the following shortest path problem:

$$
\min _{x \in \Omega} \sum_{(i, j) \in \mathcal{A}} p_{i j} c_{i j} x_{i j}
$$

where

$$
\Omega \equiv\left\{x: \sum_{(i, j) \in \mathcal{A}} x_{i j}-\sum_{(j, i) \in \mathcal{A}} x_{j i}=b_{i} \quad \forall i \in \mathcal{N}, \text { and } x_{i j} \in\{0,1\} \quad \forall(i, j) \in \mathcal{A}\right\}
$$

The parameter $b_{i}$ has the following values:

$$
b_{i}= \begin{cases}1 & \text { if } i=\text { origin } \\ -1 & \text { if } i=\text { destination } \\ 0 & \text { otherwise }\end{cases}
$$

When $p_{i j}$ and $c_{i j}$ are known, problem (1) can be solved as a regular shortest path problem. The arc costs often arise as the product of two factors as in (1). For example, in hazardous materials transportation, $p_{i j}$ and $c_{i j}$ represent the accident probability and the accident consequence, respectively. We will discuss this application to hazardous materials transportation in Section 6.

In many realistic cases, accurate estimates for the parameters $p_{i j}$ and $c_{i j}$ may be unavailable. When only one of the factors $p_{i j}$ or $c_{i j}$ is unknown and its values are confined to a set of uncertainty, a general type of robust shortest path algorithm [14] minimizes the worst case cost as follows:

$$
\min _{x \in \Omega} \max _{\tilde{c} \in \mathcal{C}} \tilde{c}^{T} x
$$

where $\mathcal{C}$ is the set of possible realizations of the uncertain cost parameter $\tilde{c}$. When $\mathcal{C}$ is a boxconstrained set or a budgeted box-constrained set, problem (2) can be solved in polynomial time [7]. On the other hand, when $\mathcal{C}$ is an ellipsoid [6,9] or a set of scenarios [14], problem (2) becomes NP-hard, in general.

In this paper, we consider the more general problem where both $p$ and $c$ vectors are uncertain and each resides in its own uncertainty set. In particular, we consider the uncertain parameters $\tilde{p}_{i j}$ and $\tilde{c}_{i j}$ as represented by the following budgeted box-constrained uncertainty sets:

$$
\begin{gathered}
\tilde{p}_{i j}=p_{i j}+q_{i j} u_{i j} \\
\tilde{c}_{i j}=c_{i j}+d_{i j} v_{i j}
\end{gathered}
$$

where $p_{i j}, q_{i j}, c_{i j}$, and $d_{i j}$ are all nonnegative, and

$$
u_{i j} \in U=\left\{u: 0 \leq u_{i j} \leq 1 \quad \forall(i, j), \quad \sum_{(i, j)} u_{i j} \leq \Gamma_{u}\right\}
$$




$$
v_{i j} \in V=\left\{v: 0 \leq v_{i j} \leq 1 \quad \forall(i, j), \quad \sum_{(i, j)} v_{i j} \leq \Gamma_{v}\right\}
$$

and $\Gamma_{u}$ and $\Gamma_{v}$ are positive integers. This structure extends Bertsimas and Sim [7]. The parameters $\Gamma_{u}$ and $\Gamma_{v}$ are called the budgets of uncertainty and reflect the risk attitude of the decision makers: the larger the budget of uncertainty, the more risk-averse the decision maker is.

In Section 2, we study properties of the robust shortest problem with the parameter model (3), and, in Section 3, we show that the robust problem of interest can be solved in polynomial time by a method based on dual variable enumeration and regular shortest path problems. In Section 4, we provide another algorithm based on enumeration that uses a $K$-shortest path finding algorithm whose worst-case complexity is exponential, but may be efficient in real cases. In Section 5, we compare our formulation with the approach of Bertsimas and Sim [7]. In Section 6, we motivate the parameter model (3) and illustrate the algorithms with an application in hazardous materials transportation.

\section{The Robust Problem}

In this paper, we consider a robust optimization model of the form:

$$
\begin{aligned}
& \min _{x \in \Omega} \max _{u \in U, v \in V} \sum_{(i, j)}\left(p_{i j}+q_{i j} u_{i j}\right)\left(c_{i j}+d_{i j} v_{i j}\right) x_{i j} \\
= & \min _{x \in \Omega}\left[\sum_{(i, j)} p_{i j} c_{i j} x_{i j}+\max _{u \in U, v \in V} \sum_{(i, j)}\left(q_{i j} c_{i j} x_{i j} u_{i j}+p_{i j} d_{i j} x_{i j} v_{i j}+q_{i j} d_{i j} x_{i j} u_{i j} v_{i j}\right)\right]
\end{aligned}
$$

We note that the inner maximization problem is a disjoint bilinear program (DBP) for any given $x$. Although the problem is not a convex optimization problem, an optimal solution exists at an extreme point [10]. While DBP is NP-hard in general [17], this special case can be solved efficiently with transformation to a linear program.

The inner problem is equivalent to: for any $x$

$$
\begin{array}{rlr}
\max _{u, v, w} & \sum_{(i, j)}\left(q_{i j} c_{i j} x_{i j} u_{i j}+p_{i j} d_{i j} x_{i j} v_{i j}+q_{i j} d_{i j} x_{i j} w_{i j}\right) \\
\text { subject to } & u_{i j} \leq 1 & \left(\rho_{i j}\right) \\
& v_{i j} \leq 1 & \left(\mu_{i j}\right) \\
& -u_{i j}+w_{i j} \leq 0 & \left(\eta_{i j}\right) \\
& -v_{i j}+w_{i j} \leq 0 & \left(\pi_{i j}\right) \\
& \sum_{(i, j)} u_{i j} \leq \Gamma_{u} & \left(\theta_{u}\right) \\
& \sum_{(i, j)} v_{i j} \leq \Gamma_{v} & \left(\theta_{v}\right)
\end{array}
$$




$$
u_{i j}, v_{i j}, w_{i j} \geq 0
$$

When $\Gamma_{u}$ and $\Gamma_{v}$ are positive integers, we can easily show that the polytope defined by the constraints of problem (6) is integral; therefore, the optimal $u, v$ and $w$ are binary.

Lemma 1. When $\Gamma_{u}$ and $\Gamma_{v}$ are integers, a solution to problem (6) is integral for any given $x$.

Proof. We may express the constraint of (6) in a matrix-vector notation:

\begin{tabular}{|c|c|c|c|c|c|}
\hline (Row Group 1) & $I_{|\mathcal{A}|}$ & & & \multirow{6}{*}[\begin{array}{l}{u}\\
{v}\\
{w}\end{array}]{} & \multirow{5}{*}{$\leq$} \\
\hline (Row Group 2) & & $I_{|\mathcal{A}|}$ & & & \\
\hline (Row Group 3) & $-I_{|\mathcal{A}|}$ & & $I_{|\mathcal{A}|}$ & & \\
\hline (Row Group 4) & & $-I_{|\mathcal{A}|}$ & $I_{|\mathcal{A}|}$ & & \\
\hline (Row 5) & $1_{|\mathcal{A}|}^{\top}$ & & & & \\
\hline (Row & & $1_{|\mathcal{A}|}^{\top}$ & & & \\
\hline
\end{tabular}

where $I_{|\mathcal{A}|}$ is a $|\mathcal{A}| \times|\mathcal{A}|$ identity matrix, $1_{|\mathcal{A}|}$ is a $|\mathcal{A}| \times 1$ vector with all elements being unity, and

$$
u=\left[u_{1}, u_{2}, \ldots, u_{|\mathcal{A}|}\right]^{\top}, \quad v=\left[v_{1}, v_{2}, \ldots, v_{|\mathcal{A}|}\right]^{\top}, \quad w=\left[w_{1}, w_{2}, \ldots, w_{|\mathcal{A}|}\right]^{\top}
$$

Empty partitions are all zero.

For any collection of rows of the constraint matrix in (7), we can construct two partitions such that the sum of rows in one partition minus the sum of rows in the other partition has only $-1,0,+1$ in each column; then it is totally unimodular $[8,11]$. That is, for any collection of rows, if we multiply +1 or -1 to each row then the sum of rows will have only $-1,0,+1$ in each column.

In any collection of rows, we first multiply +1 to rows from Row Group 3 and -1 to rows from Row Group 4. If Row 5 is present in the collection, we multiply +1 to Row 5 ; if Row 6 is present, we multiply -1 to Row 6 . Then, the current sum of rows has only $-1,0,+1$ in each column. We can multiply \pm 1 to rows from Row Groups 1 and 2 appropriately to keep $-1,0,+1$ in each column of the sum of rows. Therefore, the constraint matrix is totally unimodular.

Given that $\Gamma_{u}$ and $\Gamma_{v}$ are integers and the constraint matrix is totally unimodular, a solution of (6) is integral.

Lemma 2. Problem (6) is equivalent to the inner problem of (4).

Proof. We need to prove that $w_{i j}=u_{i j} v_{i j}$ at any optimum. For any $(i, j)$,

- If $u_{i j}=1, v_{i j}=1$, then $w_{i j} \leq 1$. Because we maximizes $q_{i j} d_{i j} x_{i j} w_{i j}$ with all coefficients nonnegative, $w_{i j}=1$ at any optimum.

- If $u_{i j}=0, v_{i j}=1$, then $w_{i j} \leq 0$, and therefore $w_{i j}=0$.

- If $u_{i j}=1, v_{i j}=0$, then $w_{i j} \leq 0$, and therefore $w_{i j}=0$. 
- If $u_{i j}=0, v_{i j}=0$, then $w_{i j} \leq 0$, and therefore $w_{i j}=0$.

Because any solution of (6) is integral by Lemma 1, this completes the proof of $w_{i j}=u_{i j} v_{i j}$.

Let us consider the dual problem of (6) with the corresponding dual variables in parentheses:

$$
\begin{array}{cl}
\min _{\theta_{u}, \theta_{v}, \rho_{i j}, \mu_{i j}, \eta_{i j}, \pi_{i j}} & \Gamma_{u} \theta_{u}+\Gamma_{v} \theta_{v}+\sum_{(i, j)}\left(\rho_{i j}+\mu_{i j}\right) \\
\text { subject to } & \rho_{i j}-\eta_{i j}+\theta_{u} \geq q_{i j} c_{i j} x_{i j} \\
& \mu_{i j}-\pi_{i j}+\theta_{v} \geq p_{i j} d_{i j} x_{i j} \\
& \eta_{i j}+\pi_{i j} \geq q_{i j} d_{i j} x_{i j} \\
& \rho_{i j}, \mu_{i j}, \eta_{i j}, \pi_{i j}, \theta_{u}, \theta_{v} \geq 0
\end{array}
$$

Using strong duality, we can write the robust optimization problem (4) as:

$$
\min \Gamma_{u} \theta_{u}+\Gamma_{v} \theta_{v}+\sum_{(i, j)}\left(p_{i j} c_{i j} x_{i j}+\rho_{i j}+\mu_{i j}\right)
$$

subject to $\quad x \in \Omega$

$$
\begin{aligned}
& \rho_{i j}-\eta_{i j}+\theta_{u} \geq q_{i j} c_{i j} x_{i j} \\
& \mu_{i j}-\pi_{i j}+\theta_{v} \geq p_{i j} d_{i j} x_{i j} \\
& \eta_{i j}+\pi_{i j} \geq q_{i j} d_{i j} x_{i j} \\
& \rho_{i j}, \mu_{i j}, \eta_{i j}, \pi_{i j}, \theta_{u}, \theta_{v} \geq 0
\end{aligned}
$$

which is a mixed integer linear program (MILP). Problem (3) can be solved by solving a finite number of shortest path problems when either $\Gamma_{u}$ or $\Gamma_{v}$ is zero [7].

\section{A Dual Variable Enumeration Approach}

In this section, we present a dual variable enumeration method, in which we search a finite number of $\left(\theta_{u}, \theta_{v}\right)$ pairs to solve problem (13). We will first represent $\rho_{i j}+\mu_{i j}$ as a function of $\theta_{u}, \theta_{v}$ and $x$ and eliminate the constraints (15)-(17). Then, we show that, in each sub-space of the whole $\left(\theta_{u}, \theta_{v}\right)$-space, we can find a solution at an extreme point; consequently, we can examine all such extreme points of sub-spaces to find an optimal $\left(\theta_{u}, \theta_{v}\right)$ pair. We will need to solve a (nominal) shortest-path problem for each extreme-point examination. Some improvements follow in Sections 3.1 and 3.2 .

The following theorem represents $\pi_{i j}, \eta_{i j}, \rho_{i j}$, and $\mu_{i j}$ using $\theta_{u}, \theta_{v}$, and $x$.

Theorem 1. For any given $\theta_{u}$ and $\theta_{v}$, there exists an optimal solution to (8)-(12) such that:

$$
\begin{aligned}
\pi_{i j} & =\min \left(q_{i j} d_{i j} x_{i j}, \max \left(\theta_{v}-p_{i j} d_{i j} x_{i j}, 0\right)\right) \\
\eta_{i j} & =q_{i j} d_{i j} x_{i j}-\min \left(q_{i j} d_{i j} x_{i j}, \max \left(\theta_{v}-p_{i j} d_{i j} x_{i j}, 0\right)\right)
\end{aligned}
$$




$$
\begin{aligned}
& \rho_{i j}=\max \left(q_{i j} c_{i j} x_{i j}+q_{i j} d_{i j} x_{i j}-\min \left(q_{i j} d_{i j} x_{i j}, \max \left(\theta_{v}-p_{i j} d_{i j} x_{i j}, 0\right)\right)-\theta_{u}, 0\right) \\
& \mu_{i j}=\max \left(p_{i j} d_{i j} x_{i j}+\min \left(q_{i j} d_{i j} x_{i j}, \max \left(\theta_{v}-p_{i j} d_{i j} x_{i j}, 0\right)\right)-\theta_{v}, 0\right)
\end{aligned}
$$

for all $(i, j) \in \mathcal{A}$.

Proof. First, we will represent the solution of (8)-(12) in terms of $\theta_{v}$ and $x$. We observe that there exists an optimal solution such that

$$
\eta_{i j}+\pi_{i j}=q_{i j} d_{i j} x_{i j} \quad \forall(i, j) \in \mathcal{A}
$$

for any given $x$. This is because minimizing $\eta_{i j}$ and $\pi_{i j}$ as much as possible may lead to smaller values of $\theta_{u}, \rho_{i j}$ and $\mu_{i j}$ which decrease the objective function value, and $\eta_{i j}$ and $\pi_{i j}$ are not present in the objective function. Therefore there exists an optimal solution such that

$$
\begin{aligned}
& 0 \leq \eta_{i j} \leq q_{i j} d_{i j} x_{i j} \\
& 0 \leq \pi_{i j} \leq q_{i j} d_{i j} x_{i j}
\end{aligned}
$$

for all $(i, j) \in \mathcal{A}$ and we can determine $\eta_{i j}$ and $\pi_{i j}$ by some allocation of $q_{i j} d_{i j} x_{i j}$ between $\eta_{i j}$ and $\pi_{i j}$ such that other constraints are satisfied.

Now suppose that $\theta_{u}$ and $\theta_{v}$ are fixed. Then we can write the constraints (9) and (10) as

$$
\begin{aligned}
\rho_{i j} & =\max \left(q_{i j} c_{i j} x_{i j}-\theta_{u}+\eta_{i j}, 0\right) \\
\mu_{i j} & =\max \left(p_{i j} d_{i j} x_{i j}-\theta_{v}+\pi_{i j}, 0\right)
\end{aligned}
$$

Increasing $\eta_{i j}$ and $\pi_{i j}$ may increase $\rho_{i j}$ and $\mu_{i j}$ and consequently increase the objective function value. Therefore, we need to find a way to allocate $q_{i j} d_{i j} x_{i j}$ to $\eta_{i j}$ and $\pi_{i j}$ without increasing the objective function value. We observe that, if $q_{i j} c_{i j} x_{i j}-\theta_{u}<0$ for some $(i, j) \in \mathcal{A}$, we can increase $\eta_{i j}$ without increasing $\rho_{i j}$. Therefore, an optimal solution in this case is to allocate $q_{i j} d_{i j} x_{i j}$ to $\eta_{i j}$ as much as we can, that is, until $q_{i j} c_{i j} x_{i j}-\theta_{u}+\eta_{i j}=0$. We can apply a similar argument to $\mu_{i j}$ and $\pi_{i j}$. On the other hand, if $q_{i j} c_{i j} x_{i j}-\theta_{u} \geq 0$ and $p_{i j} d_{i j} x_{i j}-\theta_{v} \geq 0$, then any allocation to $\eta_{i j}$ and $\pi_{i j}$ will have the same impact to the objective function value, since the cost coefficients of $\rho_{i j}$ and $\mu_{i j}$ are identical.

From the above observations, we can consider the following optimal allocation rules for $\eta_{i j}$ and $\pi_{i j}$ when $\theta_{u}$ and $\theta_{v}$ are fixed:

Case 1. For $(i, j) \in \mathcal{A}$ such that

$$
\begin{aligned}
& q_{i j} c_{i j} x_{i j}-\theta_{u} \geq 0 \\
& p_{i j} d_{i j} x_{i j}-\theta_{v} \geq 0
\end{aligned}
$$

any combination of $\eta_{i j} \geq 0$ and $\pi_{i j} \geq 0$ such that $\eta_{i j}+\pi_{i j}=q_{i j} d_{i j} x_{i j}$ is optimal. 
Case 2. For $(i, j) \in \mathcal{A}$ such that

$$
\begin{aligned}
& q_{i j} c_{i j} x_{i j}-\theta_{u}<0 \\
& p_{i j} d_{i j} x_{i j}-\theta_{v} \geq 0
\end{aligned}
$$

we can first allocate some of $q_{i j} d_{i j} x_{i j}$ to $\eta_{i j}$ until $q_{i j} c_{i j} x_{i j}-\theta_{u}+\eta_{i j}=0$ in advance, and then allocate any remaining amount to $\eta_{i j}$ and $\pi_{i j}$ by any combination. For example, we can consider $\eta_{i j}=q_{i j} d_{i j} x_{i j}$ and $\pi_{i j}=0$.

Case 3. For $(i, j) \in \mathcal{A}$ such that

$$
\begin{aligned}
q_{i j} c_{i j} x_{i j}-\theta_{u} & \geq 0 \\
p_{i j} d_{i j} x_{i j}-\theta_{v} & <0
\end{aligned}
$$

we can first allocate some of $q_{i j} d_{i j} x_{i j}$ to $\pi_{i j}$ until $p_{i j} d_{i j} x_{i j}-\theta_{v}+\pi_{i j}=0$ in advance, and then allocate any remaining amount to $\eta_{i j}$ and $\pi_{i j}$ by any combination. For example, we can consider $\pi_{i j}=q_{i j} d_{i j} x_{i j}$ and $\eta_{i j}=0$.

Case 4. For $(i, j) \in \mathcal{A}$ such that

$$
\begin{aligned}
& q_{i j} c_{i j} x_{i j}-\theta_{u}<0 \\
& p_{i j} d_{i j} x_{i j}-\theta_{v}<0
\end{aligned}
$$

we can first allocate some of $q_{i j} d_{i j} x_{i j}$ to $\eta_{i j}$ until $q_{i j} c_{i j} x_{i j}-\theta_{u}+\eta_{i j}=0$, and then allocate any remaining amount to $\pi_{i j}$ until $p_{i j} d_{i j} x_{i j}-\theta_{v}+\pi_{i j}=0$. If there is any remaining amount, we can allocate additionally to $\eta_{i j}$ and $\pi_{i j}$ by any combination.

Using the above optimal allocation rules, we can obtain an optimal solution for given $\theta_{u}$ and $\theta_{v}$. From Cases 3 and 4 , when $p_{i j} d_{i j} x_{i j}-\theta_{v}<0$, we can consider allocating $\min \left(q_{i j} d_{i j} x_{i j}, \theta_{v}-p_{i j} d_{i j} x_{i j}\right)$ to $\pi_{i j}$ without worrying about $\eta_{i j}$. From Cases 1 and 2 , when $p_{i j} d_{i j} x_{i j}-\theta_{v} \geq 0$, we can simply put $\pi_{i j}=0$. Therefore, an optimal allocation to $\pi_{i j}$ is

$$
\pi_{i j}=\min \left(q_{i j} d_{i j} x_{i j}, \max \left(\theta_{v}-p_{i j} d_{i j} x_{i j}, 0\right)\right)
$$

for all $(i, j) \in \mathcal{A}$ and any given $\theta_{v} \geq 0$. Using the condition (23), we have the corresponding allocation to $\eta_{i j}$ as

$$
\eta_{i j}=q_{i j} d_{i j} x_{i j}-\min \left(q_{i j} d_{i j} x_{i j}, \max \left(\theta_{v}-p_{i j} d_{i j} x_{i j}, 0\right)\right)
$$

for all $(i, j) \in \mathcal{A}$ and any given $\theta_{v} \geq 0$. Note that the expression (20) is not dependent on $\theta_{u}$.

From (19) and (24), we can now determine $\rho_{i j}$ and $\mu_{i j}$ as follows:

$$
\rho_{i j}=\max \left(q_{i j} c_{i j} x_{i j}-\theta_{u}+\eta_{i j}, 0\right)
$$




$$
\begin{aligned}
& =\max \left(q_{i j} c_{i j} x_{i j}+q_{i j} d_{i j} x_{i j}-\min \left(q_{i j} d_{i j} x_{i j}, \max \left(\theta_{v}-p_{i j} d_{i j} x_{i j}, 0\right)\right)-\theta_{u}, 0\right) \\
\mu_{i j} & =\max \left(p_{i j} d_{i j} x_{i j}-\theta_{v}+\pi_{i j}, 0\right) \\
& =\max \left(p_{i j} d_{i j} x_{i j}+\min \left(q_{i j} d_{i j} x_{i j}, \max \left(\theta_{v}-p_{i j} d_{i j} x_{i j}, 0\right)\right)-\theta_{v}, 0\right)
\end{aligned}
$$

We obtain the theorem.

We can now express $\rho_{i j}+\mu_{i j}$ as a function of $x_{i j}$ with cost coefficients dependent on $\theta_{u}$ and $\theta_{v}$.

Lemma 3. For any given $\theta_{u}$ and $\theta_{v}$, suppose a solution to (8)-(12) is given as in (19)-(22). The sum $\rho_{i j}+\mu_{i j}$ can be expressed as follows:

$$
\rho_{i j}+\mu_{i j}= \begin{cases}0 \cdot x_{i j} & \text { if Condition } 1 \text { or Condition } 4 \text { holds } \\ \left(q_{i j} c_{i j}-\theta_{u}\right) x_{i j} & \text { if Condition } 2 \text { holds } \\ \left(p_{i j} d_{i j}+q_{i j} c_{i j}+q_{i j} d_{i j}-\theta_{u}-\theta_{v}\right) x_{i j} & \text { if Condition } 3 \text { or Condition } 5 \text { holds } \\ \left(p_{i j} d_{i j}-\theta_{v}\right) x_{i j} & \text { if Condition } 6 \text { holds. }\end{cases}
$$

for each $(i, j) \in \mathcal{A}$ and all $\theta_{u} \geq 0$ and $\theta_{v} \geq 0$, where

Condition 1: $\theta_{u} \geq q_{i j} c_{i j}$, and $\theta_{v} \geq p_{i j} d_{i j}+q_{i j} d_{i j}$

Condition 2: $\theta_{u} \leq q_{i j} c_{i j}$, and $\theta_{v} \geq p_{i j} d_{i j}+q_{i j} d_{i j}$

Condition $3: p_{i j} d_{i j} \leq \theta_{v} \leq p_{i j} d_{i j}+q_{i j} d_{i j}$, and $\theta_{u}+\theta_{v} \leq p_{i j} d_{i j}+q_{i j} c_{i j}+q_{i j} d_{i j}$

Condition $4: p_{i j} d_{i j} \leq \theta_{v} \leq p_{i j} d_{i j}+q_{i j} d_{i j}$, and $\theta_{u}+\theta_{v} \geq p_{i j} d_{i j}+q_{i j} c_{i j}+q_{i j} d_{i j}$

Condition 5: $\theta_{u} \leq q_{i j} c_{i j}+q_{i j} d_{i j}$, and $\theta_{v} \leq p_{i j} d_{i j}$

Condition 6: $\theta_{u} \geq q_{i j} c_{i j}+q_{i j} d_{i j}$, and $\theta_{v} \leq p_{i j} d_{i j}$

Proof. For the simplicity of notation, we drop the subscript $i j$ and let $c_{0}=p c, c_{1}=p d, c_{2}=q c$, $c_{3}=q d$. We obtain

$$
\begin{aligned}
\mu & =\max \left(c_{1} x-\theta_{v}+\min \left(c_{3} x, \max \left(\theta_{v}-c_{1} x, 0\right)\right), 0\right) \\
& =\max \left(\min \left(\left(c_{1}+c_{3}\right) x-\theta_{v}, \max \left(c_{1} x-\theta_{v}, 0\right)\right),\right) \\
& = \begin{cases}\max \left(\max \left(c_{1} x-\theta_{v}, 0\right), 0\right) & \text { if }\left(c_{1}+c_{3}\right) x-\theta_{v} \geq 0 \\
\max \left(\left(c_{1}+c_{3}\right) x-\theta_{v}, 0\right) & \text { if }\left(c_{1}+c_{3}\right) x-\theta_{v} \leq 0\end{cases} \\
& = \begin{cases}\max \left(c_{1} x-\theta_{v}, 0\right) & \text { if }\left(c_{1}+c_{3}\right) x-\theta_{v} \geq 0 \\
0 & \text { if }\left(c_{1}+c_{3}\right) x-\theta_{v} \leq 0\end{cases} \\
& = \begin{cases}c_{1} x-\theta_{v} & \text { if } c_{1} x-\theta_{v} \geq 0 \\
0 & \text { if }-c_{3} x \leq c_{1} x-\theta_{v} \leq 0 \\
0 & \text { if } c_{1} x-\theta_{v} \leq-c_{3} x\end{cases}
\end{aligned}
$$




$$
\begin{aligned}
& =\max \left(c_{1} x-\theta_{v}, 0\right) \\
& =\max \left(c_{1}-\theta_{v}, 0\right) x
\end{aligned}
$$

where the last equality is due to the binarity of $x$. Again using the binarity of $x$, we can express $\rho$ as follows:

$$
\begin{aligned}
\rho & =\max \left(\left(c_{2}+c_{3}\right) x-\theta_{u}-\min \left(c_{3} x, \max \left(\theta_{v}-c_{1} x, 0\right)\right), 0\right) \\
& =\max \left(\left(c_{2}+c_{3}\right)-\theta_{u}-\min \left(c_{3}, \max \left(\theta_{v}-c_{1}, 0\right)\right), 0\right) x
\end{aligned}
$$

For three intervals of $\theta_{v}$, we obtain the following expressions of $\rho$ :

$$
\rho= \begin{cases}\max \left(c_{2}-\theta_{u}, 0\right) x & \text { if } \theta_{v} \geq c_{1}+c_{3} \\ \max \left(c_{1}+c_{2}+c_{3}-\theta_{u}-\theta_{v}, 0\right) x & \text { if } c_{1} \leq \theta_{v} \leq c_{1}+c_{3} \\ \max \left(c_{2}+c_{3}-\theta_{u}, 0\right) x & \text { if } \theta_{v} \leq c_{1}\end{cases}
$$

Therefore, we obtain the lemma.

Lemma 3 means that we can obtain an optimal solution to (13) by solving a nominal shortest path problem when $\theta_{u}$ and $\theta_{v}$ are given.

Figure 1 illustrates how the value of $\rho_{i j}+\mu_{i j}$ varies according to Lemma 3 in each region of $\left(\theta_{u}, \theta_{v}\right)$ for each $(i, j) \in \mathcal{A}$. We observe that in each shaded region of $\left(\theta_{u}, \theta_{v}\right)$, the cost coefficient of $x_{i j}$ in $\rho_{i j}+\mu_{i j}$ becomes a linear function of $\theta_{u}$ and $\theta_{v}$. We extend this observation to all links in $\mathcal{A}$.

Let $\left\{a_{k}\right\}$ be the ordered sequence of $q_{i j} c_{i j}+q_{i j} d_{i j}$ and $q_{i j} c_{i j}$ for all $(i, j) \in \mathcal{A}$ and 0 , which is the set of $\theta_{u}$-values where nonlinearlity occurs in $\rho_{i j}+\mu_{i j}$. Similarly, let $\left\{b_{l}\right\}$ be the ordered sequence of $p_{i j} d_{i j}$ and $p_{i j} d_{i j}+q_{i j} d_{i j}$ for all $(i, j) \in \mathcal{A}$ and 0 , for such $\theta_{v}$-values. Let $\left\{f_{m}\right\}$ be the ordered sequence of $p_{i j} d_{i j}+q_{i j} c_{i j}+q_{i j} d_{i j}$ for all $(i, j) \in \mathcal{A}$ and 0 , for such $\left(\theta_{u}+\theta_{v}\right)$-values.

We consider the following problem:

$$
\begin{aligned}
Z_{k l m}=\min _{x, \theta_{u}, \theta_{v}} & \Gamma_{u} \theta_{u}+\Gamma_{v} \theta_{v}+\sum_{(i, j)}\left(p_{i j} c_{i j} x_{i j}+\rho_{i j}+\mu_{i j}\right) \\
\text { subject to } \quad & x \in \Omega \\
& a_{k} \leq \theta_{u} \leq a_{k+1} \\
& b_{l} \leq \theta_{v} \leq b_{l+1} \\
& f_{m} \leq \theta_{u}+\theta_{v} \quad \text { if } f_{m} \in\left[a_{k}+b_{l}, a_{k+1}+b_{l+1}\right] \\
& f_{m+1} \geq \theta_{u}+\theta_{v} \quad \text { if } f_{m+1} \in\left[a_{k}+b_{l}, a_{k+1}+b_{l+1}\right]
\end{aligned}
$$

We note that within the $\left(\theta_{u}, \theta_{v}\right)$-space defined by $(28)-(31)$ for each tuple $(k, l, m)$, the cost coeffi- 


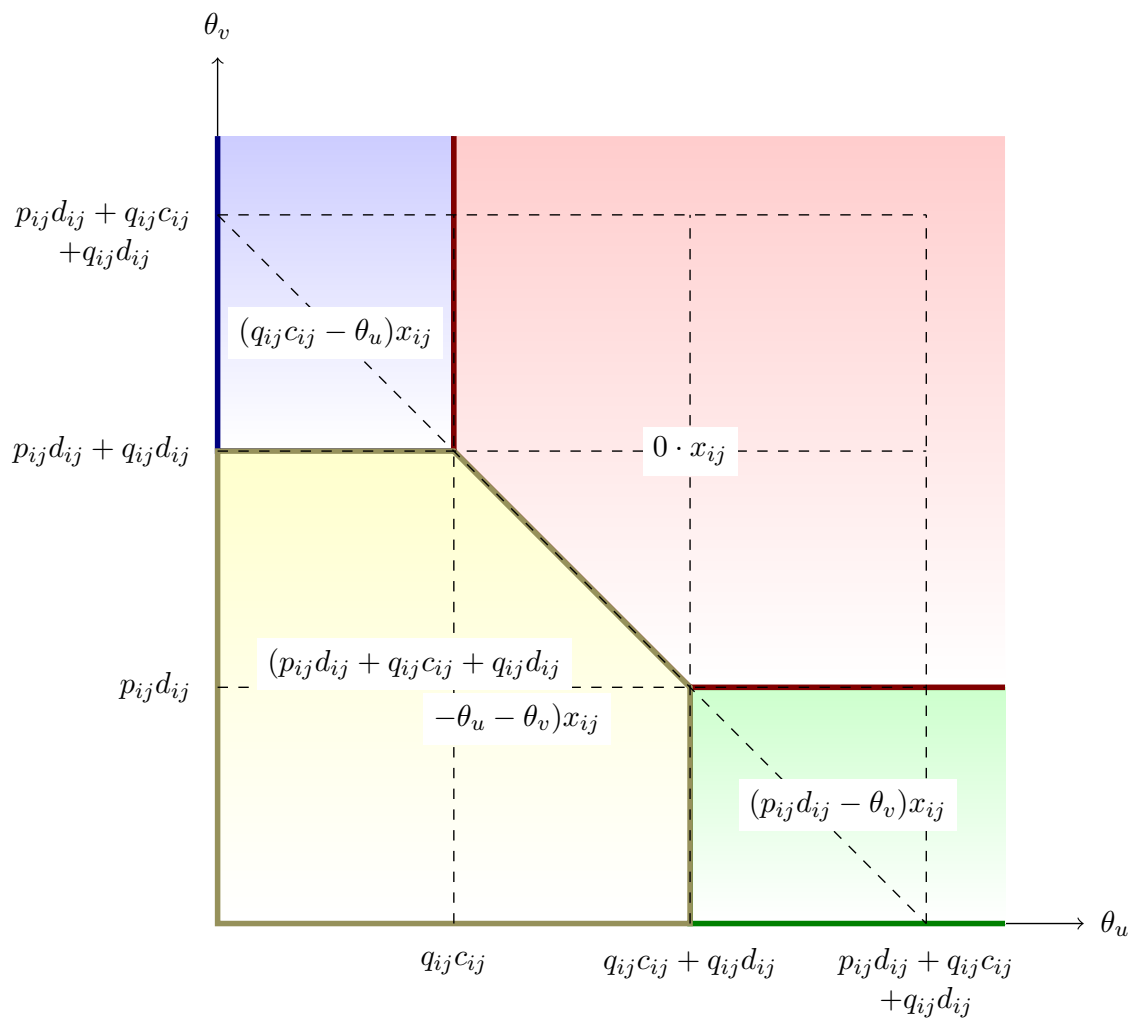

Figure 1: The value of $\rho_{i j}+\mu_{i j}$ for each interval of $\left(\theta_{u}, \theta_{v}\right)$ for each link $(i, j) \in \mathcal{A}$

cient of $x$ becomes linear in $\theta_{u}$ and $\theta_{v}$ for all links $(i, j) \in \mathcal{A}$ and for all $x$. Therefore, for problem $Z_{k l m}$, we always obtain a solution at an extreme point of the feasible space defined by (28)-(31). This idea is represented in Figure 2. For the feasible region $\mathbf{A}_{\mathbf{k l m}}$ defined by (28)-(31) in Figure 2, we know one of the six extreme points is a solution of problem (26). Therefore, we can solve problem (26) by examining those six points; for each point, the problem is a regular shortest-path problem.

If we extend this idea to the entire $\left(\theta_{u}, \theta_{v}\right)$-space, we know that we can solve the robust shortest path problem by examining the following points:

- intersections of $\theta_{u}=\left\{a_{k}\right\}$ and $\theta_{v}=\left\{b_{l}\right\}$

- intersections of $\theta_{u}=\left\{a_{k}\right\}$ and $\theta_{u}+\theta_{v}=\left\{f_{m}\right\}$

- intersections of $\theta_{v}=\left\{b_{l}\right\}$ and $\theta_{u}+\theta_{v}=\left\{f_{m}\right\}$

Accordingly, we define the following three sets of $\left(\theta_{u}, \theta_{v}\right)$ :

$$
\begin{aligned}
\Theta_{1}=\left\{\left(\theta_{u}, \theta_{v}\right): \theta_{u} \in\{0\} \cup\left\{q_{i j} c_{i j}+q_{i j} d_{i j}, q_{i j} c_{i j}:(i, j) \in \mathcal{A}\right\}\right. & \\
\theta_{v} & \left.\in\{0\} \cup\left\{p_{i j} d_{i j}, p_{i j} d_{i j}+q_{i j} d_{i j}:(i, j) \in \mathcal{A}\right\}\right\}
\end{aligned}
$$




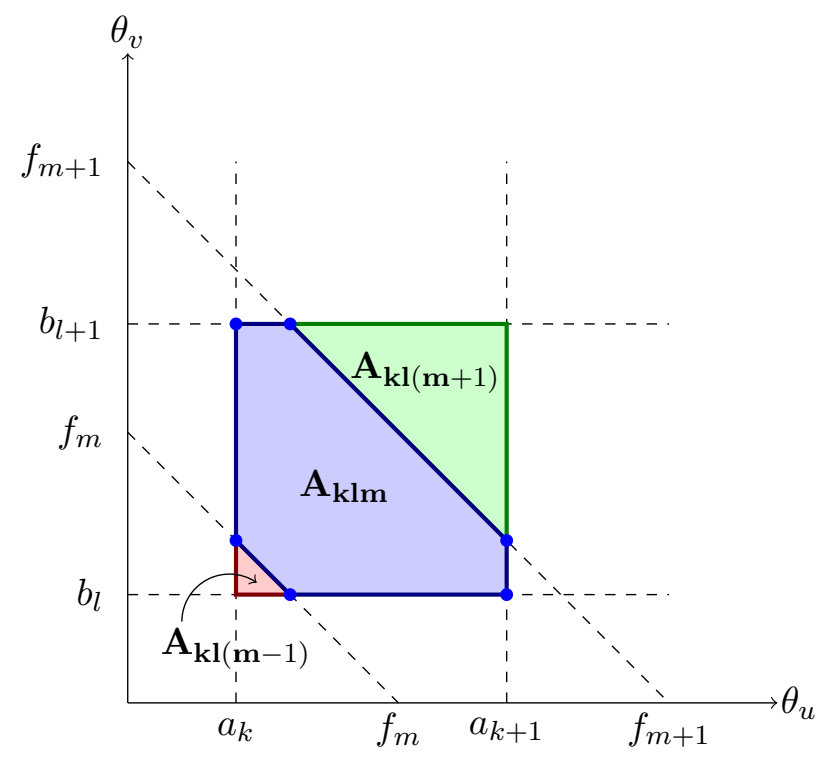

Figure 2: An illustration of decomposed feasible $\left(\theta_{u}, \theta_{v}\right)$-space on the mesh

$$
\begin{aligned}
& \Theta_{2}=\left\{\left(\theta_{u}, \theta_{v}\right): \theta_{u} \in\{0\} \cup\left\{q_{i j} c_{i j}+q_{i j} d_{i j}, q_{i j} c_{i j}:(i, j) \in \mathcal{A}\right\},\right. \\
& \left.\theta_{u}+\theta_{v} \in\{0\} \cup\left\{p_{i j} d_{i j}+q_{i j} c_{i j}+q_{i j} d_{i j}:(i, j) \in \mathcal{A}\right\}, \quad \theta_{v} \geq 0\right\} \\
& \Theta_{3}=\left\{\left(\theta_{u}, \theta_{v}\right): \theta_{v} \in\{0\} \cup\left\{p_{i j} d_{i j}, p_{i j} d_{i j}+q_{i j} d_{i j}:(i, j) \in \mathcal{A}\right\},\right. \\
& \left.\theta_{u}+\theta_{v} \in\{0\} \cup\left\{p_{i j} d_{i j}+q_{i j} c_{i j}+q_{i j} d_{i j}:(i, j) \in \mathcal{A}\right\}, \quad \theta_{u} \geq 0\right\}
\end{aligned}
$$

We obtain the following theorem:

Theorem 2. Let us define the following problem with an arbitrary constraint set $\Theta$ :

$$
Z(\Theta)=\min _{x \in \Omega,\left(\theta_{u}, \theta_{v}\right) \in \Theta} \Gamma_{u} \theta_{u}+\Gamma_{v} \theta_{v}+\sum_{(i, j)}\left(p_{i j} c_{i j} x_{i j}+\rho_{i j}+\mu_{i j}\right)
$$

Then the robust shortest path problem (3) is equivalent to the following problem:

$$
Z^{*}=\min \left\{Z\left(\Theta_{1}\right), Z\left(\Theta_{2}\right), Z\left(\Theta_{3}\right)\right\}
$$

Now we obtain the computational complexity of (33).

Theorem 3. The computational complexity of $(33)$ is $O\left(|\mathcal{N}|^{6}\right)$ and the number of shortest-path problems to be solved is $O\left(|\mathcal{N}|^{4}\right)$. 
Proof. First we note that the sizes of feasible sets are

$$
\left|\Theta_{1}\right|=O\left(|\mathcal{A}|^{2}\right), \quad\left|\Theta_{2}\right|=O\left(|\mathcal{A}|^{2}\right), \quad\left|\Theta_{3}\right|=O\left(|\mathcal{A}|^{2}\right)
$$

When we consider $|\mathcal{A}|=O\left(\mathcal{N}^{2}\right)$, the number of shortest path problems we need solve to obtain $Z^{*}$ is $O\left(|\mathcal{N}|^{4}\right)$. Since the complexity of Dijkstra's algorithm is $O\left(|\mathcal{N}|^{2}\right)$, the computational complexity of $(33)$ is $O\left(|\mathcal{N}|^{6}\right)$. Since there are other algorithms with better worst-case complexity [2], this provides an upper bound.

Obviously, the number of shortest-path problems to be solved in this method is significantly less than in the full primal variable $(u$ and $v)$ enumeration. When the full primal variable enumeration is used, we need to solve $\left(\begin{array}{c}|\mathcal{A}| \\ \Gamma_{u}\end{array}\right) \times\left(\begin{array}{c}|\mathcal{A}| \\ \Gamma_{v}\end{array}\right)$ number of shortest-path problems, which is $1.0651 \times 10^{20}$ when $|\mathcal{A}|=150, \Gamma_{u}=4$, and $\Gamma_{v}=8$. In the same network, the dual variable enumeration method solves 151,148 shortest path problems (see Section 6.1).

\subsection{An Improvement of the Dual-Variable Enumeration Approach}

In this section, we improve the dual-variable enumeration approach by reducing the number of $\left(\theta_{u}, \theta_{v}\right)$ pairs to examine, therefore reducing the number of shortest path sub-problems to solve. In particular, we will examine a subset of $\Theta_{1} \cup \Theta_{2} \cup \Theta_{3}$.

In Figure 2, the extreme points of the area $\mathbf{A}_{\mathbf{k l m}}$ are created by joining the interval diagrams like Figure 1 of two or three arcs. We observe that there are some elements of $\Theta_{1}, \Theta_{2}$, and $\Theta_{3}$ that are not created by joining the interval diagrams - they are just interior points - depending on the coefficients $p_{i j}, q_{i j}, c_{i j}$, and $d_{i j}$.

An example diagram for two $\operatorname{arcs}(i, j)$ and $(k, l)$ is provided in Figure 3. Without loss of generality, we assume that $q_{i j} c_{i j}+p_{i j} d_{i j}+q_{i j} d_{i j} \geq q_{k l} c_{k l}+p_{k l} d_{k l}+q_{k l} d_{k l}$. For arc $(i, j)$, we need to consider the following $\left(\theta_{u}, \theta_{v}\right)$ pairs (denoted by square dots on the thinner solid line):

$$
\begin{aligned}
& \left(0, p_{i j} d_{i j}+q_{i j} d_{i j}\right) \\
& \left(q_{i j} c_{i j}, p_{i j} d_{i j}+q_{i j} d_{i j}\right) \\
& \left(q_{i j} c_{i j}+q_{i j} d_{i j}, p_{i j} d_{i j}\right) \\
& \left(q_{i j} c_{i j}+q_{i j} d_{i j}, 0\right)
\end{aligned}
$$

Note that we do not need to consider $\left(q_{i j} c_{i j}+q_{i j} d_{i j}, p_{i j} d_{i j}+q_{i j} d_{i j}\right)$ and the two points at which the diagonal line meet the axes. Similarly, for arc $(k, l)$, we need to consider the four points denoted by square dots on the thicker solid line.

Two new points are created as extreme points, i.e. candidate points for the $\left(\theta_{u}, \theta_{v}\right)$ enumeration. In Figure 3, the two new points are denoted by circle dots. In this case, they are

$$
\begin{aligned}
& \left(q_{k l} c_{k l}, p_{i j} d_{i j}+q_{i j} d_{i j}\right) \\
& \left(q_{i j} c_{i j}+q_{i j} d_{i j}, p_{k l} d_{k l}\right)
\end{aligned}
$$




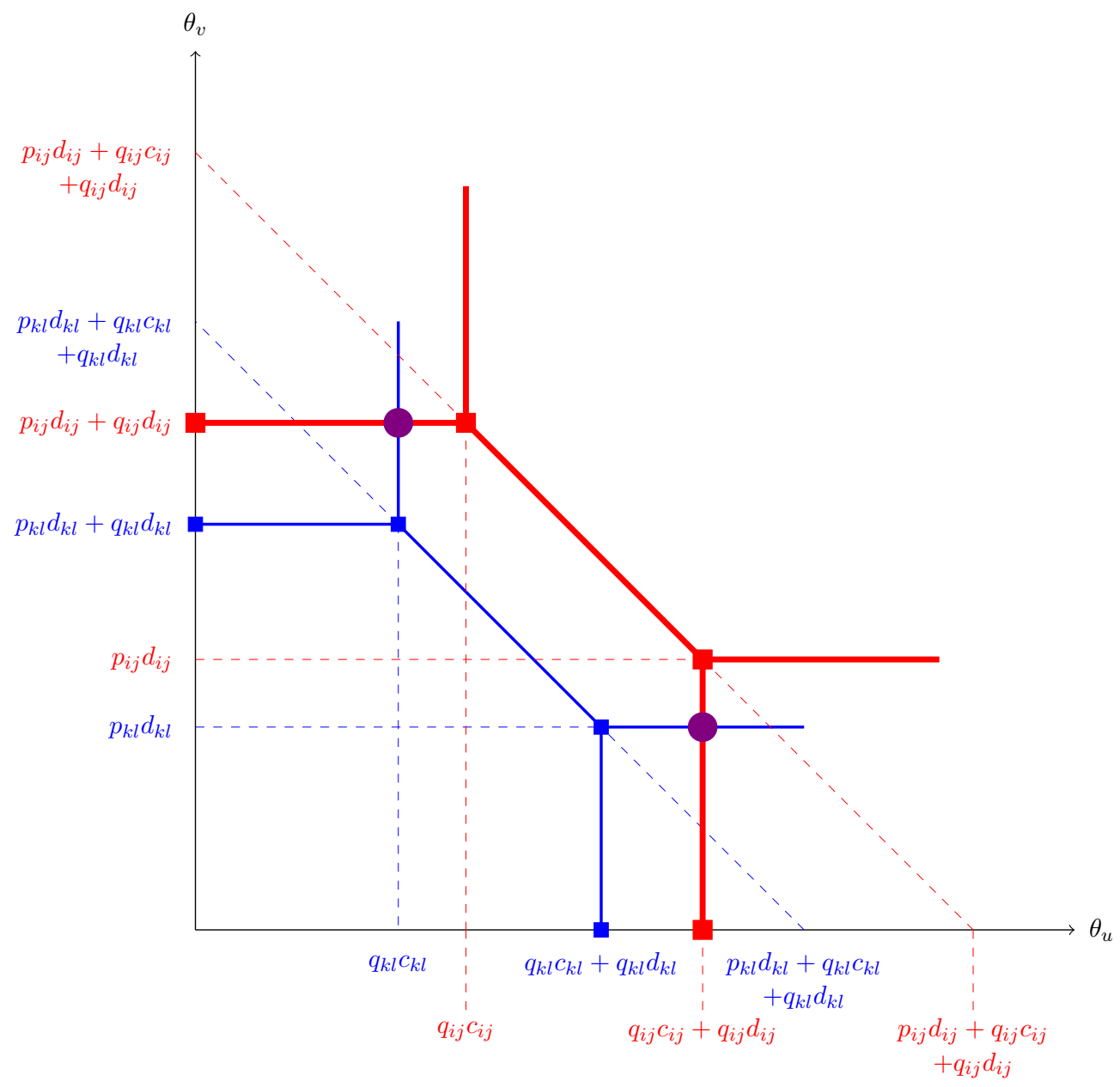

Figure 3: Joining the interval diagrams for $\operatorname{arcs}(i, j)$ and $(k, l)$. The thinner solid line represents arc $(i, j)$, and the thicker solid line represents arc $(k, l)$. Two intersection points (denoted by circle dots) represent new extreme points to be considered in the dual-variable enumeration.

When there is no overlap between the interval diagrams of any pair of arcs, they intersect at two points, creating two extreme points to consider. When there is some overlap, it creates only one or no new extreme point.

We observe that there are 11 possible cases of intersection patterns between two interval diagrams. Figure 4 represents a case of intersection patterns. Figure $4 \mathrm{a}$ is for an arc, and Figure $4 \mathrm{~b}$ is for another arc. Each line segment of the interval diagram in Figure 4a is referred by $A, B, \ldots, E$, respectively, and similarly in Figure $4 \mathrm{~b}$ by $A^{\prime}, B^{\prime}, \ldots, E^{\prime}$. Without loss of generality, we assume that $C$ is above $C^{\prime}$, i.e., the intercept of the line extending the segment $C$ is greater than $C^{\prime}$; we denote this assumption by $C_{x y}>C_{x y}^{\prime}$ (see Table 1 ).

Each line segment of the first arc can intersect with the following line segments of the second arc:

$A$ with $B^{\prime}, C^{\prime}, D^{\prime}$ 


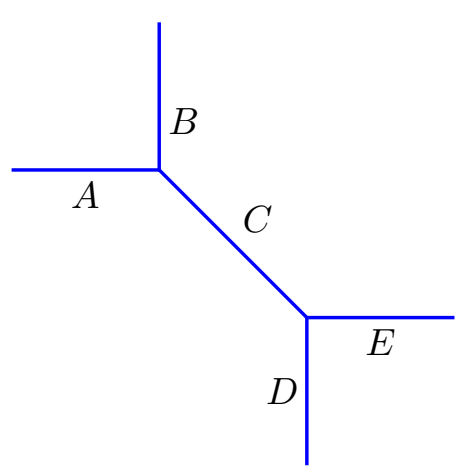

(a) A first arc

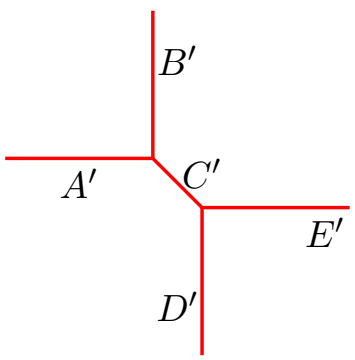

(b) A second arc

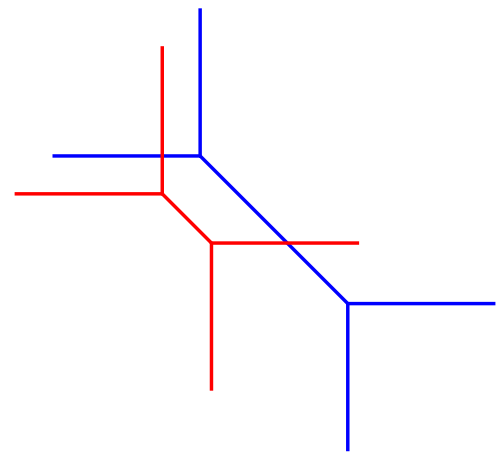

(c) A possible intersecting pattern

Figure 4: An intersecting pattern of the two interval diagrams of two arcs.

$$
\begin{aligned}
& B \text { with } E^{\prime} \\
& C \text { with } B^{\prime}, E^{\prime} \\
& D \text { with } A^{\prime}, C^{\prime}, E^{\prime} \\
& E \text { with } B^{\prime}
\end{aligned}
$$

Given the above possible intersecting pairs, we can enumerate the following 11 distinct intersecting patterns depending on the data values:

$$
\begin{array}{ll}
A \oplus B^{\prime} \text { and } C \oplus E^{\prime} & (\text { Pattern 1) } \\
A \oplus B^{\prime} \text { and } D \oplus E^{\prime} & (\text { Pattern 2) } \\
A \oplus B^{\prime} \text { and } D \oplus C^{\prime} & \text { (Pattern 3) } \\
A \oplus C^{\prime} \text { and } C \oplus E^{\prime} & (\text { Pattern 4) } \\
A \oplus C^{\prime} \text { and } D \oplus E^{\prime} & \text { (Pattern 5) } \\
A \oplus C^{\prime} \text { and } D \oplus C^{\prime} & \text { (Pattern 6) } \\
A \oplus D^{\prime} \text { and } B \oplus E^{\prime} & \text { (Pattern 7) } \\
C \oplus B^{\prime} \text { and } C \oplus E^{\prime} & \text { (Pattern 8) } \\
C \oplus B^{\prime} \text { and } D \oplus E^{\prime} & \text { (Pattern 9) } \\
C \oplus B^{\prime} \text { and } D \oplus C^{\prime} & \text { (Pattern 10) } \\
D \oplus A^{\prime} \text { and } E \oplus B^{\prime} & \text { (Pattern 11) }
\end{array}
$$

where $\oplus$ means 'intersects with'. Note that both Patterns 7 and 11 should be considered, because they are not impacted by the values of $C_{x y}$ and $C_{x y}^{\prime}$. Figure $4 \mathrm{c}$ shows an example of Pattern 1, and Figure 3 shows Pattern 2. Each pattern provides only 2 new extreme points to be considered and they are provided in Table 1 with conditions.

We construct the set of candidate $\left(\theta_{u}, \theta_{v}\right)$ pairs as following (as opposed to $\Theta_{1} \cup \Theta_{2} \cup \Theta_{3}$ ): 
Table 1: The coordinate of the new extreme points to consider for each pattern, when the first arc is $(i, j)$ (segments $A, B, C, D$, and $E)$ and the second arc is $(k, l)$ (segments $A^{\prime}, B^{\prime}, C^{\prime}, D^{\prime}$, and $\left.E^{\prime}\right) . A_{y}$ denotes the vertical coordinate $\left(\theta_{v}\right.$-axis) of the line segment $A, B_{x}$ denotes the horizontal coordinate $\left(\theta_{u}\right.$-axis) of the line segment $B, C_{x y}$ denotes the intercept-both intercepts are same of the line that extends the segment $C$, and so on. Without loss of generality, we assume $C_{x y}>C_{x y}^{\prime}$, or $p_{i j} d_{i j}+q_{i j} c_{i j}+q_{i j} d_{i j}>p_{k l} d_{k l}+q_{k l} c_{k l}+q_{k l} d_{k l}$.

\begin{tabular}{lllcl}
\hline Patterns & Intersections & Conditions & New Extreme Points to Consider, $\left(\theta_{u}, \theta_{v}\right)$ \\
\hline Pattern1 & $A \oplus B^{\prime}$ and $C \oplus E^{\prime}$ & $A_{y}>A_{y}^{\prime}, B_{x}>B_{x}^{\prime}, E_{y}<E_{y}^{\prime}$ & $\left(B_{x}^{\prime}, A_{y}\right), \quad\left(C_{x y}-E_{y}^{\prime}, E_{y}^{\prime}\right)$ \\
\hline Pattern2 & $A \oplus B^{\prime}$ and $D \oplus E^{\prime}$ & $A_{y}>A_{y}^{\prime}, B_{x}>B_{x}^{\prime}, E_{y}>E_{y}^{\prime}, D_{x}>D_{x}^{\prime}$ & $\left(B_{x}^{\prime}, A_{y}\right), \quad\left(D_{x}, E_{y}^{\prime}\right)$ \\
\hline Pattern3 & $A \oplus B^{\prime}$ and $D \oplus C^{\prime}$ & $A_{y}>A_{y}^{\prime}, B_{x}>B_{x}^{\prime}, D_{x}<D_{x}^{\prime}, E_{y}>E_{y}^{\prime}$ & $\left(B_{x}^{\prime}, A_{y}\right),\left(D_{x}, C_{x y}^{\prime}-D_{x}\right)$ \\
\hline Pattern4 & $A \oplus C^{\prime}$ and $C \oplus E^{\prime}$ & $E_{y}^{\prime}<A_{y}<A_{y}^{\prime}, B_{x}>B_{x}^{\prime}, E_{y}<E_{y}^{\prime}$ & $\left(C_{x y}^{\prime}-A_{y}, A_{y}\right), \quad\left(C_{x y}-E_{y}^{\prime}, E_{y}^{\prime}\right)$ \\
\hline Pattern5 & $A \oplus C^{\prime}$ and $D \oplus E^{\prime}$ & $E_{y}^{\prime}<A_{y}<A_{y}^{\prime}, B_{x}>B_{x}^{\prime}, E_{y}>E_{y}^{\prime}, D_{x}>D_{x}^{\prime}$ & $\left(C_{x y}^{\prime}-A_{y}, A_{y}\right),\left(D_{x}, E_{y}^{\prime}\right)$ \\
\hline Pattern6 & $A \oplus C^{\prime}$ and $D \oplus C^{\prime}$ & $E_{y}^{\prime}<A_{y}<A_{y}^{\prime}, B_{x}>B_{x}^{\prime}, D_{x}<D_{x}^{\prime}, E_{y}>E_{y}^{\prime}$ & $\left(C_{x y}^{\prime}-A_{y}, A_{y}\right),\left(D_{x}, C_{x y}^{\prime}-D_{x}\right)$ \\
\hline Pattern7 & $A \oplus D^{\prime}$ and $B \oplus E^{\prime}$ & $B_{x}>D_{x}^{\prime}, A_{y}<E_{y}^{\prime}$ & $\left(D_{x}^{\prime}, A_{y}\right),\left(B_{x}, E_{y}^{\prime}\right)$ \\
\hline Pattern8 & $C \oplus B^{\prime}$ and $C \oplus E^{\prime}$ & $\left.B_{x}<B_{x}^{\prime}, E_{y}<E_{y}^{\prime}-B_{x}^{\prime}\right), \quad\left(C_{x y}-E_{y}^{\prime}, E_{y}^{\prime}\right)$ \\
\hline Pattern9 & $C \oplus B^{\prime}$ and $D \oplus E^{\prime}$ & $B_{x}<B_{x}^{\prime}, E_{y}>E_{y}^{\prime}, D_{x}>D_{x}^{\prime}$ & $\left(B_{x}^{\prime}, C_{x y}-B_{x}^{\prime}\right), \quad\left(D_{x}, E_{y}^{\prime}\right)$ \\
\hline Pattern10 & $C \oplus B^{\prime}$ and $D \oplus C^{\prime}$ & $B_{x}<B_{x}^{\prime}, D_{x}<D_{x}^{\prime}, E_{y}>E_{y}^{\prime}$ & $\left(B_{x}^{\prime}, C_{x y}-B_{x}^{\prime}\right), \quad\left(D_{x}, C_{x y}^{\prime}-D_{x}\right)$ \\
\hline Pattern11 & $D \oplus A^{\prime}$ and $E \oplus B^{\prime}$ & $\left.E_{y}>A_{y}^{\prime}, D_{x}<B_{x}^{\prime}\right), \quad\left(B_{x}^{\prime}, E_{y}\right)$ \\
\hline \hline
\end{tabular}


Step 0. Order the set of arcs in the descending order of $p_{i j} d_{i j}+q_{i j} c_{i j}+q_{i j} d_{i j}:|\mathcal{A}|=\left\{a_{1}, a_{2}, \ldots, a_{|\mathcal{A}|}\right\}$, where $a_{n}$ denotes $n$-th arc. Set $\Theta \leftarrow\{(0,0)\}$, and $t \leftarrow 1$.

Step 1. Denoting the $t$-th arc by $(i, j)$, add the following pairs to the set $\Theta$ :

$$
\begin{aligned}
& \left(0, p_{i j} d_{i j}+q_{i j} d_{i j}\right) \\
& \left(q_{i j} c_{i j}, p_{i j} d_{i j}+q_{i j} d_{i j}\right) \\
& \left(q_{i j} c_{i j}+q_{i j} d_{i j}, p_{i j} d_{i j}\right) \\
& \left(q_{i j} c_{i j}+q_{i j} d_{i j}, 0\right)
\end{aligned}
$$

Step 2. Consider each arc $a_{s}$ in $\left\{a_{t+1}, \ldots, a_{|\mathcal{A}|}\right\}$, denoting it by $(k, l)$. Examine the intersecting pattern between $(i, j)$ and $(k, l)$ according to the conditions provided in Table 1 , and add the corresponding two new extreme points to the set $\Theta$. If equality holds for conditions, ignore; the corresponding point is already added, or will be added later.

Note that, in Step 2, we always have $C_{x y}>C_{x y}^{\prime}$, because of the ordering in Step 0 .

Theorem 4. The maximum size of the resulting set $\Theta$ in the improvement proposed in Steps 0 to 2 as above is

$$
|\mathcal{A}|^{2}+3|\mathcal{A}|+1
$$

which is also the maximum number of shortest path sub-problems to solve.

Proof. In Step 0, we add one element, $(0,0)$. For each arc in $\mathcal{A}$, we add four elements in Step 1; therefore we add $4|\mathcal{A}|$ elemtents. For each pair from $\mathcal{A}$, we add two elements in Step 2, that is, we add 2 elements for $\frac{|\mathcal{A}|(|\mathcal{A}|-1)}{2}$ pairs. Therefore, we obtain

$$
1+4|\mathcal{A}|+2 \frac{|\mathcal{A}|(|\mathcal{A}|-1)}{2}=|\mathcal{A}|^{2}+3|\mathcal{A}|+1
$$

If there are some extreme points that coincide with others, the total number is less than (34).

Once the set $\Theta$ is determined, the optimal value is determined by:

$$
Z^{*}=\min _{\left(\theta_{u}, \theta_{v}\right) \in \Theta} \min _{x \in \Omega} \Gamma_{u} \theta_{u}+\Gamma_{v} \theta_{v}+\sum_{(i, j)}\left(p_{i j} c_{i j} x_{i j}+\rho_{i j}+\mu_{i j}\right)
$$

where $\rho_{i j}+\mu_{i j}$ values are determined as in (25).

Although the order of computational complexity of the improved dual variable enumeration approach is same as the original - still $O\left(|\mathcal{A}|^{2}\right)$ or $O\left(|\mathcal{N}|^{4}\right)$ — the improved approach solves significantly smaller number of sub problems. We demonstrate this point with examples later. 


\subsection{Another Improvement of the Dual-Variable Enumeration Approach}

While searching the sets of $\Theta_{1}, \Theta_{2}$, and $\Theta_{3}$, we shall record the current minimum value, $z^{\sharp}$, of the objective function value

$$
\Gamma_{u} \theta_{u}+\Gamma_{v} \theta_{v}+\sum_{(i, j)}\left(p_{i j} c_{i j} x_{i j}+\rho_{i j}+\mu_{i j}\right)
$$

When we encounter a next $\left(\theta_{u}, \theta_{v}\right)$ pair, we first examine if we need to solve the corresponding shortest path sub-problem. If

$$
\Gamma_{u} \theta_{u}+\Gamma_{v} \theta_{v} \geq z^{\sharp}
$$

then we do not need to solve the shortest path sub-problem for the current $\left(\theta_{u}, \theta_{v}\right)$ dual variable pair. All coefficients $p, q, c$ and $d$ are nonnegative - recall that we assumed so - therefore the optimal objective function value of the shortest path sub-problem is nonnegative. Consequently, if the condition (36) is met, the current $\left(\theta_{u}, \theta_{v}\right)$ cannot yield a better objective function value than the objective function value $z^{\sharp}$.

With this improvement, we can save computing time by missing many shortest path subproblems to solve, but we cannot generally quantify how many shortest path sub-problems we can skip. In an application in Section 6, this technique is shown to be very effective in saving computing time.

In summary, an improved form of the dual-variable enumeration approach can be stated as follows:

Step 0. Determine the set $\Theta$ as in Section 3.1. Set $z^{\sharp} \leftarrow \infty$ and $k \leftarrow 1$.

Step 1. Let $\left(\theta_{u}^{k}, \theta_{v}^{k}\right)$ be the $k$-th element of $\Theta$. If

$$
\Gamma_{u} \theta_{u}^{k}+\Gamma_{v} \theta_{v}^{k} \geq z^{\sharp}
$$

go to Step 3.

Step 2 For $\left(\theta_{u}^{k}, \theta_{v}^{k}\right)$, by solving a shortest-path problem, compute the following:

$$
z^{k}=\Gamma_{u} \theta_{u}^{k}+\Gamma_{v} \theta_{v}^{k}+\sum_{(i, j)}\left(p_{i j} c_{i j} x_{i j}+\rho_{i j}^{k}+\mu_{i j}^{k}\right)
$$

where $\rho_{i j}^{k}+\mu_{i j}^{k}$ is determined as in (25) for $\left(\theta_{u}^{k}, \theta_{v}^{k}\right)$. If $z^{k}<z^{\sharp}$, set $z^{\sharp} \leftarrow z^{k}$.

Step 3. Update $k \leftarrow k+1$, and repeat Steps 1 and 2 until $k=|\mathcal{A}|$.

\section{A Path Enumeration Approach}

In this section, we provide another algorithm whose worst-case complexity is exponential, but may be efficient in many real cases. Let us denote the nominal shortest path by $l_{1}$ and its corresponding 
objective function value and $x$-value by $z_{1}$ and $x^{1}$, respectively. That is,

$$
z_{1}=\min _{x \in \Omega} \sum p_{i j} c_{i j} x_{i j}=\sum p_{i j} c_{i j} x_{i j}^{1}
$$

The corresponding maximum possible path cost is obtained by

$$
z_{1}^{R}=\max _{u \in U, v \in V} \sum\left(p_{i j}+q_{i j} u_{i j}\right)\left(c_{i j}+d_{i j} v_{i j}\right) x_{i j}^{1}
$$

which can be solved as a linear program; see (6). Similarly, we define $z_{k}$ and $z_{k}^{R}$ for any path $l_{k}$. Then, we note that the following relationship holds:

$$
z_{k} \leq z_{k}^{R} \quad \forall l_{k}
$$

The objective of the robust problem is to find a path $l_{*}$ that attains $z_{*}^{R}=\min _{l_{k} \in \mathcal{P}} z_{k}^{R}$. After solving the nominal shortest path problem (37), we know

$$
z_{*}^{R} \leq z_{1}^{R}
$$

Therefore, any path $l_{k}$ whose nominal path cost is greater than $z_{k}^{R}$ is not a solution to the robust problem, because for such path $l_{k}$, we have $z_{*}^{R} \leq z_{1}^{R}<z_{k} \leq z_{k}^{R}$.

Let us now define the set of all paths whose nominal objective function value $\left(z_{k}\right)$ is less than or equals to $z_{1}^{R}$

$$
\mathcal{P}_{c}=\left\{l_{1}, l_{2}, \ldots, l_{\left|\mathcal{P}_{c}\right|}\right\}
$$

We know that the set $\mathcal{P}_{c}$ contains the robust path. Therefore, once we have the set $\mathcal{P}_{c}$, computing $z_{k}^{R}$ for all paths in the set requires solving $\left|\mathcal{P}_{c}\right|$ linear programs, whose dimension is as small as three times the number of arcs contained in each path $l_{k}$.

To determine the set $\mathcal{P}_{c}$, we can use any $K$-shortest paths finding algorithm that provides paths in the ascending order of path length and allows termination at any point when the path length exceeds a certain value. Therefore the complexity of finding the set $\mathcal{P}_{c}$ depends on the size of the set and the complexity of finding $K$-shortest paths. For example, we can use Yen's algorithm [18] whose complexity is $O(K|\mathcal{N}|(|\mathcal{A}|+|\mathcal{N}| \log |\mathcal{N}|))$. However, the number $K$ for our algorithm is unknown a priori, and in the worst case $K$ is equal to the number of all available paths; therefore the worst case complexity becomes exponential.

We can further reduce the computational effort by stopping the algorithm as soon as the nominal path cost exceeds the minimum value of $z_{k}^{R}$ among the paths found so far. That is, when we construct the set $\mathcal{P}_{c}$, we update the reference cost value by the current value of $\min _{l_{j} \in \mathcal{P}_{c}} z_{j}^{R}$. The algorithm is summarized as follows:

Step 0. Find the nominal shortest path $l_{1}$ by solving a shortest path problem, and obtain the worst-case path cost $z_{1}^{R}$ by solving the $3\left|l_{1}\right|$-dimensional linear program of the form (6). Set $k=1, z^{R}=z_{1}^{R}$ and $l^{*}=l_{1}$. 
Step 1. Find the next best nominal shortest path $l_{k+1}$ with $z_{k+1} \leq z^{R}$. If no such path exists, stop. $l^{*}$ is the optimal robust path.

Step 2. Obtain the worst-path cost $z_{k+1}^{R}$ by solving the $3\left|l_{k+1}\right|$-dimensional linear program of the form (6). If $z_{k+1}^{R} \leq z^{R}$ then set $z^{R}=z_{k+1}^{R}$ and $l^{*}=l_{k+1}$. Set $k=k+1$. Go to Step 1 and repeat.

This path enumeration algorithm is shown to be more efficient than the dual variable enumeration method when the level of uncertainty is small in Table 8.

\section{Comparison with the Existing Approach}

We can compare our robust problem (4) with the "regular" approach of Bertsimas and Sim [7] considering a single uncertain cost vector. Let us define the single uncertain cost vector $\tilde{m}_{i j}=\tilde{p}_{i j} \tilde{c}_{i j}$ so that

$$
\tilde{m}_{i j} \in\left[p_{i j} c_{i j},\left(p_{i j}+q_{i j}\right)\left(c_{i j}+d_{i j}\right)\right] \equiv\left[m_{i j}, m_{i j}+n_{i j}\right]
$$

In addition we define $z^{U V}\left(\Gamma_{u}, \Gamma_{v}\right)$ to denote the optimal objective function value of the proposed approach in (4) with budgets of uncertainty $\Gamma_{u}$ and $\Gamma_{v}$, and $z^{W}\left(\Gamma_{w}\right)$ to denote the optimal objective function value of the regular approach with the single uncertain cost vector in (38) with the single budget of uncertainty $\Gamma_{w}$. That is,

$$
\begin{aligned}
z^{U V}\left(\Gamma_{u}, \Gamma_{v}\right) & =\min _{x \in \Omega} \max _{u \in U, v \in V} \sum_{(i, j)}\left(p_{i j}+q_{i j} u_{i j}\right)\left(c_{i j}+d_{i j} v_{i j}\right) x_{i j} \\
z^{W}\left(\Gamma_{w}\right) & =\min _{x \in \Omega} \max _{w \in W} \sum_{(i, j)}\left(m_{i j}+n_{i j} w_{i j}\right) x_{i j}
\end{aligned}
$$

where

$$
W=\left\{w: 0 \leq w_{i j} \leq 1 \quad \forall(i, j), \quad \sum_{(i, j)} w_{i j} \leq \Gamma_{w}\right\}
$$

When the regular approach is used, a challenge is how to determine the budget $\Gamma_{w}$. Determining $\Gamma_{u}$ and $\Gamma_{v}$ is easier, because we could directly observe the data source for each data type to determine the budgets of uncertainty. On the other hand, the new single parameter $m$ is manipulated after data collection. Therefore, it is not obvious how we should determine how uncertain the new manipulated data is. We may determine $\Gamma_{w}=\Gamma_{u}=\Gamma_{v}$; then we always have $z^{W}\left(\Gamma_{w}\right) \leq z^{U V}\left(\Gamma_{u}, \Gamma_{v}\right)$. That is, the worst-case may not be captured by the regular approach; therefore, we need the proposed approach to consider the real worst-case even in the special case. We further provide the following results without proof:

Lemma 4. Depending on the budgets of uncertainty, we can compare the proposed approach with the regular approach [7] as follows:

1. If $\Gamma_{w} \leq \min \left(\Gamma_{u}, \Gamma_{v}\right)$, we always have $z^{W}\left(\Gamma_{w}\right) \leq z^{U V}\left(\Gamma_{u}, \Gamma_{v}\right)$. 
2. If $\Gamma_{w}=\Gamma_{u}=\Gamma_{v}$, we always have $z^{W}\left(\Gamma_{w}\right) \leq z^{U V}\left(\Gamma_{u}, \Gamma_{v}\right)$.

3. If $\Gamma_{w}=\Gamma_{u}+\Gamma_{v}$, we always have $z^{W}\left(\Gamma_{w}\right) \geq z^{U V}\left(\Gamma_{u}, \Gamma_{v}\right)$.

Proof. Let $f(x, u, v)=\sum_{(i, j)}\left(p_{i j}+q_{i j} u_{i j}\right)\left(c_{i j}+d_{i j} v_{i j}\right) x_{i j}$ and $g(x, w)=\sum_{(i, j)}\left(m_{i j}+n_{i j} w_{i j}\right) x_{i j}$, then

$$
\begin{aligned}
z^{U V}\left(\Gamma_{u}, \Gamma_{v}\right) & =\min _{x \in \Omega} \max _{u \in U, v \in V} f(x, u, v) \\
z^{W}\left(\Gamma_{w}\right) & =\min _{x \in \Omega} \max _{w \in W} g(x, w)
\end{aligned}
$$

1. $\left(\Gamma_{w} \leq \min \left(\Gamma_{u}, \Gamma_{v}\right)\right)$ For a certain $x \in \Omega$, let $\check{w}=\arg \max _{w \in W} g(x, w)$. Let $\check{u}=\check{w}$ and $\check{v}=\check{w}$. Then $\check{u} \in U$ and $\check{v} \in V$ with $\Gamma_{w} \leq \min \left(\Gamma_{u}, \Gamma_{v}\right)$. Therefore we obtain

$$
\max _{w \in W} g(x, w) \leq \max _{u \in U, v \in V} f(x, u, v) \quad \forall x \in \Omega
$$

Let $(\bar{x}, \bar{w})$ be a solution to $(40)$, i.e. $z^{W}\left(\Gamma_{w}\right)=g(\bar{x}, \bar{w})$. Then

$$
z^{W}\left(\Gamma_{w}\right) \leq \max _{w \in W} g(x, w) \leq \max _{u \in U, v \in V} f(x, u, v) \quad \forall x \in \Omega
$$

and consequently,

$$
z^{W}\left(\Gamma_{w}\right) \leq \min _{x \in \Omega} \max _{u \in U, v \in V} f(x, u, v)=z^{U V}\left(\Gamma_{u}, \Gamma_{v}\right)
$$

which completes proof for the case $\Gamma_{w}=\Gamma_{u}=\Gamma_{v}$.

2. $\left(\Gamma_{w}=\Gamma_{u}=\Gamma_{v}\right)$ This is a special case of $\Gamma_{w} \leq \min \left(\Gamma_{u}, \Gamma_{v}\right)$.

3. $\left(\Gamma_{w}=\Gamma_{u}+\Gamma_{v}\right)$ This case can be similarly proved. For a certain $x \in \Omega$, let $(\check{u}, \check{v})=$ $\arg \max _{u \in U, v \in V} f(x, u, v)$. Let $\check{w}=\max (\check{u}, \check{v})$ where 'max' operation is taken for each element, then $\check{w} \in W$ with $\Gamma_{w}=\Gamma_{u}+\Gamma_{v}$. Therefore we obtain

$$
\max _{w \in W} g(x, w) \geq \max _{u \in U, v \in V} f(x, u, v) \quad \forall x \in \Omega
$$

Let $(\bar{x}, \bar{u}, \bar{v})$ be a solution to $(39)$, i.e. $z^{U V}\left(\Gamma_{u}, \Gamma_{v}\right)=f(\bar{x}, \bar{u}, \bar{v})$. Then

$$
z^{U V}\left(\Gamma_{u}, \Gamma_{v}\right) \leq \max _{u \in U, v \in V} f(x, u, v) \leq \max _{w \in W} g(x, w) \quad \forall x \in \Omega
$$

Thus,

$$
z^{U V}\left(\Gamma_{u}, \Gamma_{v}\right) \leq \min _{x \in \Omega} \max _{w \in W} g(x, w)=z^{W}\left(\Gamma_{w}\right)
$$

This completes the proof. 

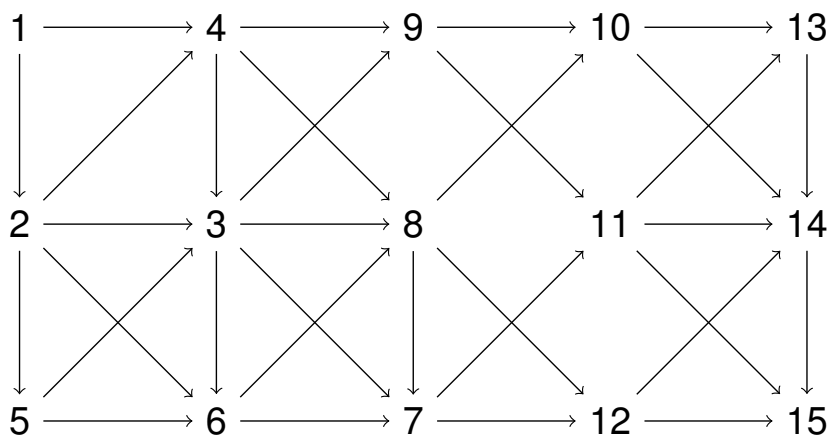

Figure 5: An abstract test network with 15 nodes and 33 links.

In the first and second cases of Lemma 4 , one would determine $\Gamma_{w}$ at the minimum budget of $\Gamma_{u}$ and $\Gamma_{v}$, but the worst-case cannot be captured with the regular approach. Setting $\Gamma_{w}=$ $\Gamma_{u}+\Gamma_{v}$ as in the third case would capture the worst-case with the regular approach, but in general it leads to unnecessarily conservative results. Therefore, one may choose $\Gamma_{w}$ in the interval of $\left[\min \left(\Gamma_{u}, \Gamma_{v}\right), \Gamma_{u}+\Gamma_{v}\right]$, but a proper choice is ambiguous to make.

\subsection{Abstract Example}

We also show, using an example, that the model of Bertsimas and Sim [7] may not be proper when the two cost coefficients are uncertain independently. We use an abstract network presented in Figure 5. The test network consists of 15 nodes and 33 links with randomly generated cost coefficients in Table 2. We used Node 1 and Node 15 as origin and destination, respectively.

We intend to compare the performance of the nominal shortest path, the robust shortest path by the Bertsimas-Sim (B-S) model [7] with a single cost vector, and the robust shortest path proposed in this paper. We used $\Gamma_{u}=2$ and $\Gamma_{v}=3$ for the proposed model, and we tested $\Gamma=1,2, \ldots, 9$ for the B-S model. The obtained paths are presented in Table 3.

Note that the B-S model cannot provide the path $l^{*}$, as seen in Table 3 . The path $l^{*}$ is the solution of the model proposed in this paper. Therefore, if the uncertainty of two costs coefficients acts indeed independently, the B-S model cannot provide an optimal robust path with any budget of uncertainty, $\Gamma$, although the worst-case cost of $l_{1}, \ldots, l_{3}$ is not significantly larger than the worst-case cost of $l^{*}$ in this example.

However, we cannot directly compare the performances of the B-S model and our model, since they are just different models for different settings. We also cannot directly compare the performances of the B-S paths with different budgets of uncertainty. For example, the path $l_{5}$ is optimally robust with $\Gamma=5$, while the path $l_{4}$ is with $\Gamma=4$. Although $l_{4}$ seems more robust to smaller uncertainties than $l_{5}$, it is not true. When $\Gamma=2$, the worst-case costs of $l_{4}$ and $l_{5}$ are 24,521 and 22,897, respectively (Table 4 ).

We do not intend to conclude which is a better robust model; rather, we want to point out that an optimal, robust path is specific to the network structure, the cost coefficient values, and 
Table 2: Cost coefficients used for the test network

\begin{tabular}{l|rrrr||c|rrrr}
\hline$(i, j)$ & $p_{i j}$ & $q_{i j}$ & $c_{i j}$ & $d_{i j}$ & $(i, j)$ & $p_{i j}$ & $q_{i j}$ & $c_{i j}$ & $d_{i j}$ \\
\hline$(1,4)$ & 79 & 31 & 66 & 28 & $(7,11)$ & 79 & 54 & 23 & 3 \\
$(1,2)$ & 59 & 97 & 41 & 93 & $(7,12)$ & 10 & 37 & 35 & 43 \\
$(2,4)$ & 31 & 21 & 50 & 40 & $(8,7)$ & 95 & 71 & 85 & 56 \\
$(2,3)$ & 90 & 52 & 95 & 38 & $(8,10)$ & 0 & 95 & 16 & 64 \\
$(2,5)$ & 9 & 23 & 95 & 59 & $(8,12)$ & 30 & 38 & 16 & 3 \\
$(2,6)$ & 32 & 57 & 73 & 7 & $(9,10)$ & 5 & 69 & 51 & 71 \\
$(3,9)$ & 89 & 100 & 38 & 21 & $(9,11)$ & 44 & 60 & 60 & 17 \\
$(3,8)$ & 66 & 13 & 4 & 72 & $(10,13)$ & 79 & 78 & 16 & 59 \\
$(3,6)$ & 68 & 95 & 58 & 58 & $(10,14)$ & 91 & 59 & 64 & 61 \\
$(3,7)$ & 47 & 12 & 56 & 20 & $(11,14)$ & 53 & 38 & 84 & 77 \\
$(4,3)$ & 14 & 19 & 36 & 84 & $(11,15)$ & 80 & 85 & 78 & 6 \\
$(4,9)$ & 95 & 65 & 88 & 42 & $(11,13)$ & 56 & 23 & 26 & 85 \\
$(4,8)$ & 88 & 13 & 62 & 54 & $(12,15)$ & 75 & 80 & 31 & 38 \\
$(5,3)$ & 44 & 8 & 62 & 53 & $(12,14)$ & 1 & 100 & 18 & 40 \\
$(5,6)$ & 83 & 66 & 30 & 19 & $(13,14)$ & 48 & 28 & 45 & 33 \\
$(6,7)$ & 33 & 3 & 7 & 8 & $(14,15)$ & 25 & 71 & 33 & 56 \\
$(6,8)$ & 37 & 99 & 29 & 46 & & & & & \\
\hline
\end{tabular}

Table 3: Comparison of Various Paths

\begin{tabular}{ccclr}
\hline Description & Path Name & Setting & Path & Worst-Case Cost $^{\mathrm{b}}$ \\
\hline Nominal & $l_{0}$ & $\Gamma=0$ & $\{1,2,4,3,8,12,14,15\}$ & 37,016 \\
\hline & $l_{1}$ & $\Gamma=1$ & $\{1,4,3,8,12,14,15\}$ & 25,616 \\
B-S $^{\mathrm{a}}$ & $l_{2}$ & $\Gamma=2$ & $\{1,4,3,8,12,14,15\}$ & 25,616 \\
& $l_{3}$ & $\Gamma=3$ & $\{1,4,3,8,12,14,15\}$ & 25,616 \\
& $l_{4}$ & $\Gamma=4$ & $\{1,4,3,7,12,15\}$ & 25,697 \\
& $l_{5}$ & $\Gamma=5$ & $\{1,4,3,8,12,15\}$ & 27,035 \\
& $l_{6}$ & $\Gamma=6$ & $\{1,4,3,8,12,15\}$ & 27,035 \\
\hline This Paper & $l^{*}$ & $\Gamma_{u}=2, \Gamma_{v}=3$ & $\{1,4,3,7,12,14,15\}$ & 25,314 \\
\hline
\end{tabular}

a Bertsimas and Sim [7]

$\mathrm{b}$ The worst-case cost measured with the uncertainty set with $\Gamma_{u}=2$ and $\Gamma_{v}=3$. 
Table 4: Comparison of Worst-Case Costs in Various Settings for Paths in Table 3

\begin{tabular}{|c|c|c|c|c|c|}
\hline & $l_{0}$ & $l_{1}, l_{2}, l_{3}$ & $l_{4}$ & $l_{5}, l_{6}$ & $l^{*}$ \\
\hline$\Gamma=0^{\mathrm{a}}$ & 6,060 & 7,305 & 11,025 & 8,787 & 9,543 \\
\hline$\Gamma=1^{\mathrm{b}}$ & 24,545 & $15,024^{\mathrm{c}}$ & 19,395 & 17,157 & 17,262 \\
\hline$\Gamma=2$ & 32,264 & 20,864 & 24,521 & 22,897 & 23,102 \\
\hline$\Gamma=3$ & 38,104 & 26,604 & 27,977 & 28,023 & 28,228 \\
\hline$\Gamma=4$ & 43,844 & 31,730 & 31,293 & 31,479 & 31,684 \\
\hline$\Gamma=5$ & 47,300 & 35,186 & 33,145 & 32,291 & 35,000 \\
\hline$\Gamma_{u}=1, \Gamma_{v}=1^{\mathrm{d}}$ & 24,545 & 15,024 & 19,395 & 17,157 & 17,262 \\
\hline$\Gamma_{u}=1, \Gamma_{v}=2$ & 29,297 & 19,776 & 21,607 & 21,909 & 19,474 \\
\hline$\Gamma_{u}=2, \Gamma_{v}=1$ & 26,888 & 17,070 & 21,441 & 19,203 & 19,308 \\
\hline$\Gamma_{u}=1, \Gamma_{v}=3$ & 30,697 & 21,988 & 22,783 & 24,121 & 20,650 \\
\hline$\Gamma_{u}=2, \Gamma_{v}=2$ & 32,264 & 21,822 & 24,521 & 955 & 23,102 \\
\hline$\Gamma_{u}=3, \Gamma_{v}=1$ & 28,688 & 18,870 & 22,736 & 19,887 & 21,108 \\
\hline$\Gamma_{u}=1, \Gamma_{v}=4$ & & & 23,723 & & 21,590 \\
\hline$\Gamma_{u}=2, \Gamma_{v}=3$ & 37,016 & 25,616 & 25,697 & 27 & 25,314 \\
\hline$\Gamma_{u}=3, \Gamma_{v}=2$ & 34,064 & 23,622 & 25,816 & 24,943 & 25,148 \\
\hline$\Gamma_{u}=4, \Gamma_{v}=1$ & 29,738 & 19,554 & 23,420 & 20,495 & 22,403 \\
\hline & & & & & \\
\hline$\Gamma_{u}=2, \Gamma_{v}=4$ & 38,256 & 27,828 & 26,637 & 28,211 & 26,490 \\
\hline$\Gamma_{u}=3, \Gamma_{v}=3$ & 38,816 & 27,662 & 27,977 & 28,023 & 28,228 \\
\hline$\Gamma_{u}=4, \Gamma_{v}=2$ & 35,114 & 24,610 & 26,500 & 25,627 & 26,443 \\
\hline$\Gamma_{u}=5, \Gamma_{v}=1$ & 30,422 & 20,162 & 24,092 & 20,547 & 23,087 \\
\hline$\Gamma_{u}=2, \Gamma_{v}=5$ & 39,432 & 29,004 & 27,067 & 28,301 & 27,430 \\
\hline$\Gamma_{u}=3, \Gamma_{v}=4$ & 42,856 & 30,742 & 29,013 & 30,491 & 29,404 \\
\hline$\Gamma_{u}=4, \Gamma_{v}=3$ & 39,866 & 28,650 & 29,272 & 28,707 & 29,523 \\
\hline$\Gamma_{u}=5, \Gamma_{v}=2$ & 35,798 & 25,294 & 27,172 & 26,235 & 27,127 \\
\hline$\Gamma_{u}=3, \Gamma_{v}=5$ & 44,096 & 31,918 & 29,953 & 30,581 & 30,344 \\
\hline$\Gamma_{u}=4, \Gamma_{v}=4$ & 43,906 & 31,730 & 31,293 & 31,479 & 31,684 \\
\hline$\Gamma_{u}=5, \Gamma_{v}=3$ & 40,854 & 29,334 & 29,944 & 29,315 & 30,207 \\
\hline$\Gamma_{u}=4, \Gamma_{v}=5$ & 46,312 & 34,198 & 32,233 & 31,569 & 32,720 \\
\hline$\Gamma_{u}=5, \Gamma_{v}=4$ & 44,894 & 32,414 & 31,965 & 32,087 & 32,979 \\
\hline$\Gamma_{u}=5, \Gamma_{v}=5$ & 47,362 & 35,186 & 33,145 & 32,291 & 35,000 \\
\hline
\end{tabular}

a Deterministic (nominal) cost.

${ }^{b}$ Worst-case costs are computed with the uncertainty set of Bertsimas and Sim [7] with the corresponding $\Gamma$.

c The minimum value in each row is bold faced.

${ }^{\mathrm{d}}$ Worst-case costs are computed with the uncertainty set of this paper with the corresponding $\Gamma_{u}$ and $\Gamma_{v}$. 
Table 5: Comparison of computing times for the abstract example

\begin{tabular}{lcccccc}
\hline & Nominal & B-S & Dual-0 $^{\mathrm{a}}$ & Dual-1 $^{\mathrm{b}}$ & Dual-2 $^{\mathrm{c}}$ & Path-Enum $^{\mathrm{d}}$ \\
\hline Computing Time (sec) & 0.008 & 0.019 & 1.787 & 0.290 & 0.230 & 4.759 \\
No. of Shortest-Path Problems & 1 & 34 & 7850 & 1189 & 916 & - \\
No. of Paths & - & - & - & - & - & 188 \\
\hline
\end{tabular}

a The dual variable enumeration approach, without any improvement

$\mathrm{b}$ The dual variable enumeration approach, with the first improvement

c The dual variable enumeration approach, with the first and second improvements

$\mathrm{d}$ The path enumeration approach

the choice of the budget of uncertainty. However, in some applications, it may be unclear how to choose a combined, single budget of uncertainty $(\Gamma)$ for the multiplication of two cost coefficients, as opposed to two separated budgets of uncertainty $\left(\Gamma_{u}\right.$ and $\left.\Gamma_{v}\right)$. We will discuss this point in depth later in this paper. In addition, the performance also depends on what measure we use. A performance test may lead to a different result with a different measure, for example, mean, variance, value-at-risk, etc.

For this test network, the computation times are provided in Table 5. We note that the two improvements in the dual variable enumeration approach reduce the number of shortest-path subproblems from 7,850 to 915. Algorithms were implemented with MATLAB and executed in a generic PC.

\section{An Application to Hazardous Materials Transportation}

Accidents involving hazardous materials (hazmat) are low-probability, high-consequence incidents. While the probability of hazmat accidents is very low, the consequences can be catastrophic. The U.S. had about 15,000 hazmat accidents in the year 1998 only 429 of which were classified as serious accidents [13]. There are two important facts that make hazmat problems a proper application of the proposed robust optimization approach. First, historical data sufficient to construct a stochastic distribution are rarely available in realistic problems. Hazmat accident probabilities are hard to obtain because hazmat accidents, especially serious ones, are rare events. In real routing decisionmaking, the accident probabilities of general traffic are used to estimate the accident probabilities of hazmat trucks, but the two kinds of probabilities might be very different. We cannot know if they are different or the same, due to the lack of available data. In addition, the consequences of hazmat accidents depend on weather conditions like wind speed and direction, the number of people present at the time of an accident, the effectiveness of evacuation, the seriousness of the accident, etc. Therefore, the impacted number of people at a hazmat accident is very difficult to estimate and subject to uncertainty. Again, historical data is not usually available for consequences.

Second, the sources of the two types of data - probabilities and consequences - are different. Accident probabilities are obtained from organizations like the U.S. Department of Transportation 
and its sub-divisions including the Pipeline and Hazardous Materials Safety Administration and the Federal Emergency Management Agency. Accident consequence data may be computed based on population information and travel pattern information available through the U.S. Census Bureau and the U.S. Commodity Flow Survey by the U.S. Bureau of Transportation Statistics. Therefore it is hard to determine a single budget of uncertainty for the regular approach with a single uncertain cost vector [7] as described in Section 5.

In addition, it is unclear if the two data are correlated. One might think that the congestion level would be high in the links with high consequence level, and the accident probability is an increasing function of the congestion level; hence, there exists positive correlation between the accident probability and the accident. However, Martin [16] observed that accident rates are highest when traffic is lightest and lowest when traffic level is modest. It is found that the accident rate decreases and then increases as the traffic volume increases. In light traffic, the accident rate is higher on weekends, while it is higher on weekdays in heavy traffic. Lord et al. [15] observed that the relationship between accident rates and traffic flow cannot be described fully without considering vehicle density, level of service, vehicle occupancy, volume/capacity ratio, and speed distribution. In addition, these studies are for all vehicles, not exclusively for hazmat vehicles. The relationship between the accident probability of hazmat vehicles and the hazmat accident consequence is hard to study, because of lack of historical data. This indicates that it is unclear how the uncertainty of the accident probability and the accident consequence can be modeled as a single data type.

Although we used population data as the measure of the accident consequence for illustration purpose in the subsequent section, depending on how the consequence is measured, the relationship varies. In an uncongested road with low population, the accident consequence by the population measure is small. However, if a nuclear power plant is located nearby, a hazmat accident would bring unwanted catastrophe. Therefore, the relationship between the accident probability and the accident consequence also depends on the measure of consequence and it is unclear what the nature of correlation between two data is, if it exists.

Suppose that we have some estimates of hazmat accident probability and accident consequence, denoted by $p_{i j}$ and $c_{i j}$, respectively, in each road segment $(i, j)$. The expected consequence of a hazmat truck traveling along path $l$ is as follows [4]:

$$
R^{l}=\sum_{\left(i_{k}, j_{k}\right) \in \mathcal{A}_{l}} \prod_{\left(i_{h}, j_{h}\right) \in \mathcal{A}_{l}, h<k}\left(1-p_{i_{h} j_{h}}\right) p_{i_{k} j_{k}} c_{i_{k} j_{k}}
$$

where $\mathcal{A}_{l}$ is the set of all arcs in path $l$, and $\left(i_{k}, j_{k}\right)$ is the $k$-th arc in path $l$. The expression (47) assumes that the shipment terminates once an accident happens in any road segment. It is noted that accident probabilities $p_{i j}$ are extremely small, usually in the range of $10^{-8}$ to $10^{-6}$ per mile traveled [1]. Therefore we can approximate as

$$
\prod_{\left(i_{h}, j_{h}\right) \in \mathcal{A}_{l}, h<k}\left(1-p_{i_{h} j_{h}}\right) \approx 1
$$


Consequently, we obtain the following approximation [12]:

$$
R^{l} \approx \sum_{(i, j) \in \mathcal{A}_{l}} p_{i j} c_{i j}
$$

The resulting nominal problem to minimize the expected consequence is a shortest path of the form (1).

Due to lack of data, the accident probability $p_{i j}$ is subject to uncertainty. In addition, the accident consequence $c_{i j}$ is also hard to estimate. In many hazmat accidents, especially those involving explosives or poisonous gas, the weather conditions are important factors [3]. One needs to consider uncertain factors involving weather conditions to determine the safest path in hazmat transportation. However, accurate weather conditions can be difficult to obtain and the resulting accident consequences may be computed as interval data at best. Therefore, the robust optimization model (4) is a natural approach to minimize the expected consequence in the worst-case scenario in hazmat transportation.

\subsection{Numerical Results}

We provide numerical results of the proposed algorithms based on Albany, New York, USA and its nearby highway network. The transportation network considered consists of 90 nodes and 150 links as presented in Figure 6.

The nominal accident probabilities are computed by $p_{i j}=10^{-6} \times($ length of link $(i, j))$ as in Abkowitz and Cheng [1]. The nominal accident consequences $c_{i j}$ are computed using the $\lambda$ neighborhood concept developed by Batta and Chiu [5]. The road length and population statistics are obtained from Department of Transportation and Department of Commerce websites. We generated $q_{i j}$ and $d_{i j}$ randomly, but in the same order as the nominal coefficients, $p_{i j}$ and $c_{i j}$. We used $\Gamma_{u}=4$ and $\Gamma_{v}=8$. While the randomly generated uncertain intervals do not represent any real scenario, our intention is to show that considering two multiplicative uncertain cost coefficients as a single coefficient may not capture the worst-case properly, and the robustness may not be proportional to the single budget of uncertainty with such consideration.

We find paths from origin node 1 to destination node 12. The nominal shortest path is

$$
\mathrm{NSP}=\{1,70,45,13,81,72,73,69,66,67,68,41,29,30,12\}
$$

the B-S robust shortest path with $\Gamma=4$ is

$$
\mathrm{BS} 4=\{1,70,45,13,14,15,81,72,73,63,52,51,50,49,48,47,40,41,29,30,12\}
$$

the B-S robust shortest path with $\Gamma=8$ is

$$
\mathrm{BS} 8=\{1,70,45,13,14,15,81,72,73,69,66,67,68,41,29,30,12\}
$$




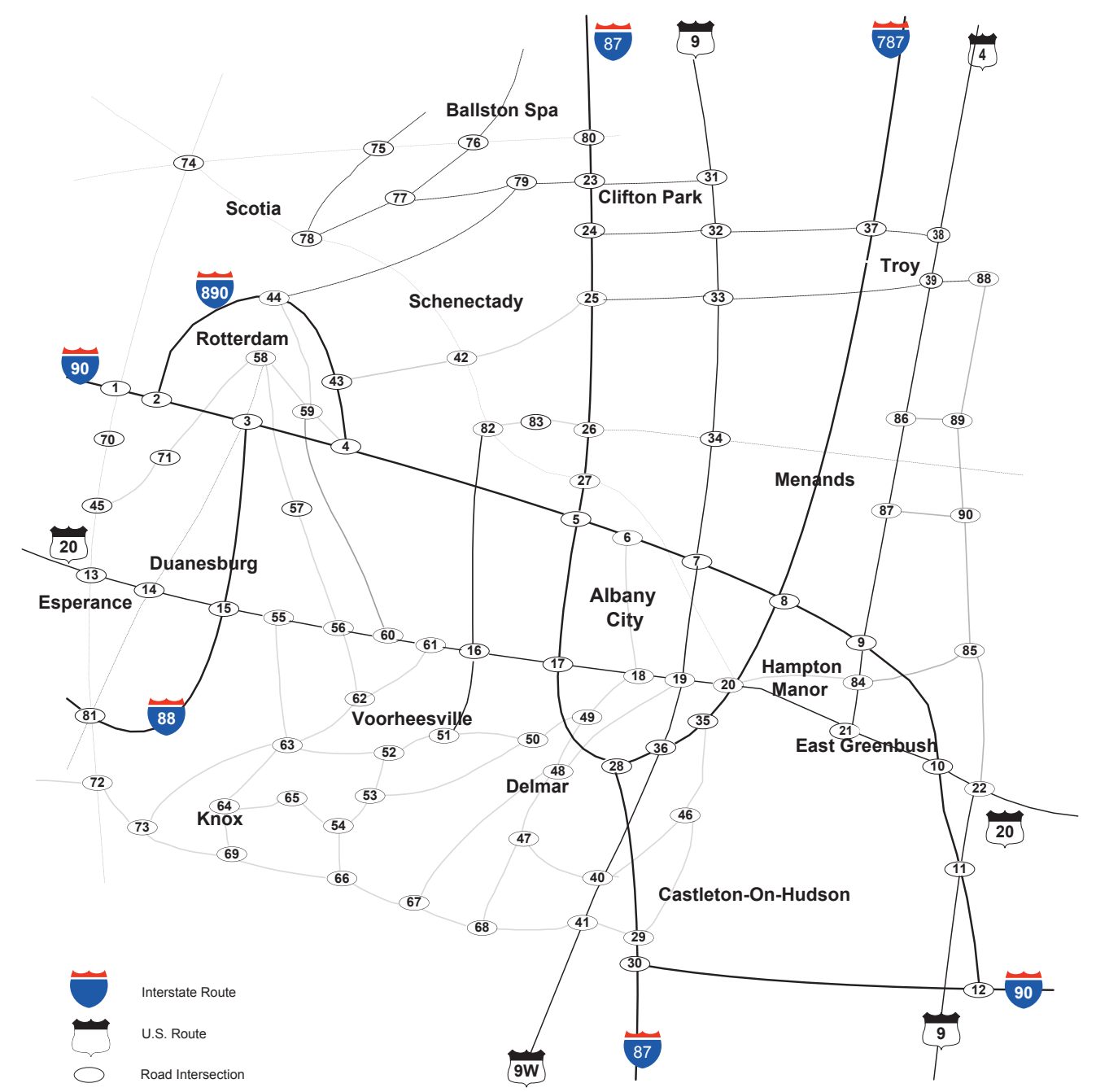

Figure 6: Albany Area Highway Network (90 nodes, 150 links) 
Table 6: Comparison of computing times for the Albany network

\begin{tabular}{lcccccc}
\hline & Nominal & B-S & Dual-0 & Dual-1 & Dual-2 & Path-Enum \\
\hline Computing Time (sec) & 0.013 & 0.106 & 95.737 & 13.923 & 3.769 & 150.389 \\
No. of Shortest Path Problems & 1 & 151 & 151,148 & 22,951 & 6,056 & - \\
No. of Paths & - & - & - & - & - & 1,323 \\
\hline
\end{tabular}
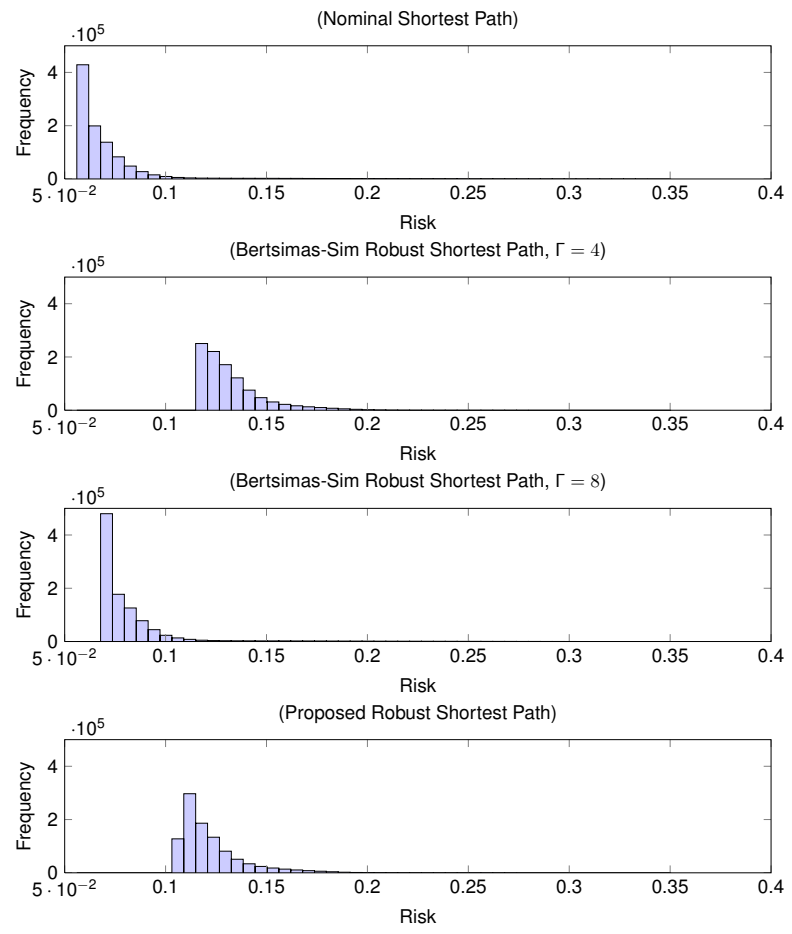

Figure 7: Histogram of the Realized Path Costs (1,000,000 samples)

and the proposed robust shortest path with $\Gamma_{u}=4$ and $\Gamma_{v}=8$ is

$$
\mathrm{KLB} 48=\{1,70,45,13,81,72,73,63,52,51,50,49,48,47,40,41,29,30,12\}
$$

(KLB is from the initials of the authors' last names.) The Dijkstra's algorithm for the nominal shortest path took 0.013 seconds, and the B-S robust shortest paths with the corresponding algorithm [7] were obtained after 0.106 seconds of computing time. For the proposed robust shortest path computation, the dual variable enumeration method (with the two improvements) took 3.769 seconds after solving 6,056 shortest path problems, and the path enumeration method took 150.389 seconds after finding 1,323 paths. The computing time is summarized in Table 6 . We used MATLAB at a generic PC running Windows 7.

To test the path performances of the above four paths, we randomly allocate the budget of uncertainty $\left(\Gamma_{u}=4, \Gamma_{v}=8\right)$, to $\left\{u_{i j}\right\}$ and $\left\{v_{i j}\right\}$, independently from each other. Although the worst-case happens when $\left\{u_{i j}\right\}$ and $\left\{v_{i j}\right\}$ are binary, we allowed any value between 0 and 1 in the simulation. We generated 1,000,000 samples and the histograms of path-costs are presented for all 

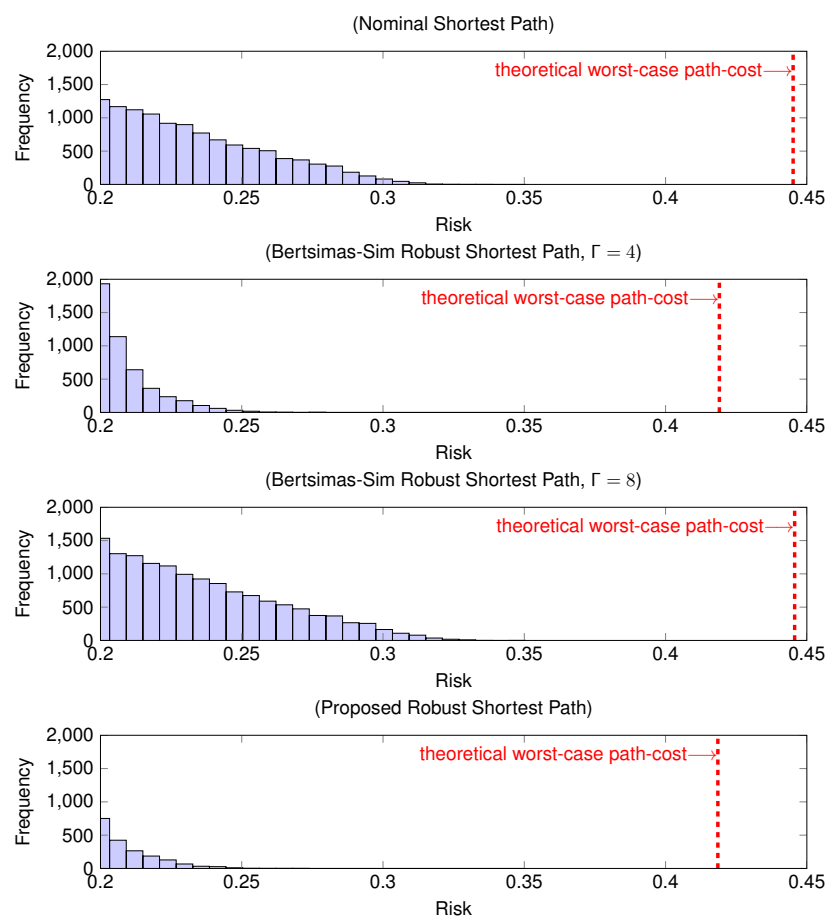

Figure 8: Closer look to histogram of the realized path costs

four paths in Figure 7. In the simulation, we assumed the link costs are distributed uniformly within the uncertain intervals, $\tilde{p}_{i j} \in\left[p_{i j}, p_{i j}+q_{i j}\right]$ and $\tilde{c}_{i j} \in\left[c_{i j}, c_{i j}+d_{i j}\right]$. Since the budget of uncertainty was allocated to links that are not included in the above four paths in many realizations, the far left path-costs in the histogram represent the nominal path-costs in the four paths. The NSP and BS8 paths show better nominal performances than the BS4 and KLB48 paths. However, higher path-costs, which are of interest in the robust optimization framework, show different patterns.

To observe path performances in the long tail, we provide a closer look to the histograms in Figure 8. The KLB48 path shows the best performance in the long-tail, which should not be a surprise since the KLB48 path is supposed so. One interesting observation in Figure 8 is that the BS8 path is weak to uncertainty and its performance is even worse than the NSP and BS4 paths. Using dashed lines, we also provide the theoretical worst-case path-costs, which the KLB48 path minimizes. The BS4 path is close to the KLB48 path in terms of the theoretical worst-case cost (the path selections are similar), and is better than the BS8 path.

A detailed summary is provided in Table 7. To compare the worst-case performances in other scenarios, we provide the theoretical worst-case path-costs in three cases: (1) treating two coefficients as a single coefficient with $\Gamma=4$, denoted by one $(\Gamma=4),(2)$ same treatment but with $\Gamma=8$, denoted by one $(\Gamma=8)$, (3) two coefficients separately as proposed in this paper, denoted by two $\left(\Gamma_{u}=4, \Gamma_{v}=8\right)$. While the theoretical worst-case path-costs do not differ very much among the four paths in the first two cases, the third case exhibits significant differences. This indicates that when more accurate robust path selection is necessary, considering two coefficients as a single coefficient may lead to non-robust path selection. In the later part in Table 7, we provide the worst- 
case, mean, and variance from the simulation results, which are consistent with the observations already explained.

Table 7: Summary of theoretical worst-case path-costs and the simulation results

\begin{tabular}{crrrr}
\hline & $\begin{array}{r}\text { Nominal Path } \\
\text { NSP }\end{array}$ & $\begin{array}{r}\text { B-S Path } \\
(\Gamma=4) \\
\text { BS4 }\end{array}$ & $\begin{array}{r}\text { B-S Path } \\
(\Gamma=8) \\
\text { BS8 }\end{array}$ & $\begin{array}{r}\text { Proposed Path } \\
\left(\Gamma_{u}=4, \Gamma_{v}=8\right) \\
\text { KLB48 }\end{array}$ \\
\hline Theoretical worst-case path-cost & & & 0.2386 \\
$(\Gamma=4)^{\mathrm{a}}$ & 0.2443 & 0.2380 & 0.2428 & 0.2849 \\
$(\Gamma=8)$ & 0.2843 & 0.2828 & 0.2821 & 0.4185 \\
$\left(\Gamma_{u}=4, \Gamma_{v}=8\right)^{\mathrm{b}}$ & 0.4451 & 0.4190 & 0.4457 & 0.2697 \\
\hline Summary of realized path-costs $(1,000,000$ samples $) ;\left(\Gamma_{u}=4, \Gamma_{v}=8\right)$ \\
\hline \multicolumn{6}{c}{ worst-case } \\
mean & 0.3379 & 0.2793 & 0.3475 & 0.1220 \\
variance $\left(\times 10^{-4}\right)$ & 0.0714 & 0.1323 & 0.0817 & 2.4000 \\
\hline
\end{tabular}

a $(\Gamma)$ means when two cost coefficients are treated as a single cost coefficient as in the B-S model.

${ }^{\mathrm{b}}\left(\Gamma_{u}, \Gamma_{v}\right)$ means when two cost coefficients are treated as proposed in this paper.

We also compare the computing performance of the dual variable enumeration (with improvements) and the path enumeration methods. For the test purpose, we set

$$
\left.\begin{array}{c}
\Gamma_{u}=\Gamma_{v}=\tau \\
q_{i j}=0.1 \times \tau \times p_{i j} \\
d_{i j}=0.1 \times \tau \times c_{i j}
\end{array}\right\} \quad \text { for } \tau=1,2, \ldots, 25
$$

in the Albany network. As $\tau$ increases, the intervals of uncertainty become bigger, and therefore the number of paths to be found in the path enumeration method increases. Until $\tau$ is 6 , the path enumeration method outperforms the dual variable enumeration method, but for $\tau$ bigger than 6 , the dual variable enumeration method finds the optimal solution faster. The computation time and the number of paths found are reported in Table 8 and Figure 9. We also note that the computation time save is significant with the two improvements for the dual variable enumeration approach.

\section{Conclusions}

The robust shortest path problem considered in this paper has the cost coefficients as multiplications of two uncertain parameters. We have shown that the problem can be solved by a dual variable enumeration method and a path enumeration method, both of which are exact algorithms. The dual variable enumeration method requires solving a finite number of shortest path sub-problems. The path enumeration method generates paths using a $K$-shortest paths algorithm, and it may be 
Table 8: Comparison of Dual Variable Enumeration Approaches and Path Enumeration Approach

\begin{tabular}{r|rr|rr|rr|rr}
\hline & \multicolumn{2}{|c|}{ Dual-0 } & \multicolumn{2}{c|}{ Dual-1 } & \multicolumn{2}{c}{ Dual-2 } & \multicolumn{2}{c}{ Path-Enum } \\
$\tau$ & Time $^{\mathrm{a}}$ & No. SPP & Time & No. SPP & Time & No. SPP & Time & No. Path \\
\hline 1 & 108.952 & 147,493 & 16.936 & 22,951 & 16.999 & 22,951 & 0.112 & 1 \\
2 & 109.408 & 147,545 & 17.017 & 22,951 & 15.766 & 21,313 & 0.214 & 2 \\
3 & 110.256 & 147,607 & 16.956 & 22,951 & 13.980 & 18,803 & 0.674 & 7 \\
4 & 109.978 & 147,691 & 17.045 & 22,951 & 11.563 & 15,511 & 1.965 & 20 \\
5 & 110.108 & 147,761 & 17.042 & 22,951 & 9.893 & 13,111 & 3.634 & 35 \\
6 & 110.517 & 147,867 & 17.227 & 22,951 & 8.546 & 11,319 & 8.047 & 69 \\
7 & 110.406 & 147,931 & 17.047 & 22,951 & 7.365 & 9,755 & 13.552 & 112 \\
8 & 110.423 & 148,051 & 17.135 & 22,951 & 6.294 & 8,315 & 22.131 & 168 \\
9 & 110.025 & 148,129 & 17.092 & 22,951 & 5.320 & 7,033 & 29.117 & 226 \\
10 & 109.803 & 148,211 & 17.110 & 22,951 & 4.457 & 5,903 & 38.039 & 326 \\
\hline
\end{tabular}

${ }^{a}$ computation time measure in seconds

${ }^{\mathrm{b}}$ number of shortest path problems solved

${ }^{c}$ number of paths found

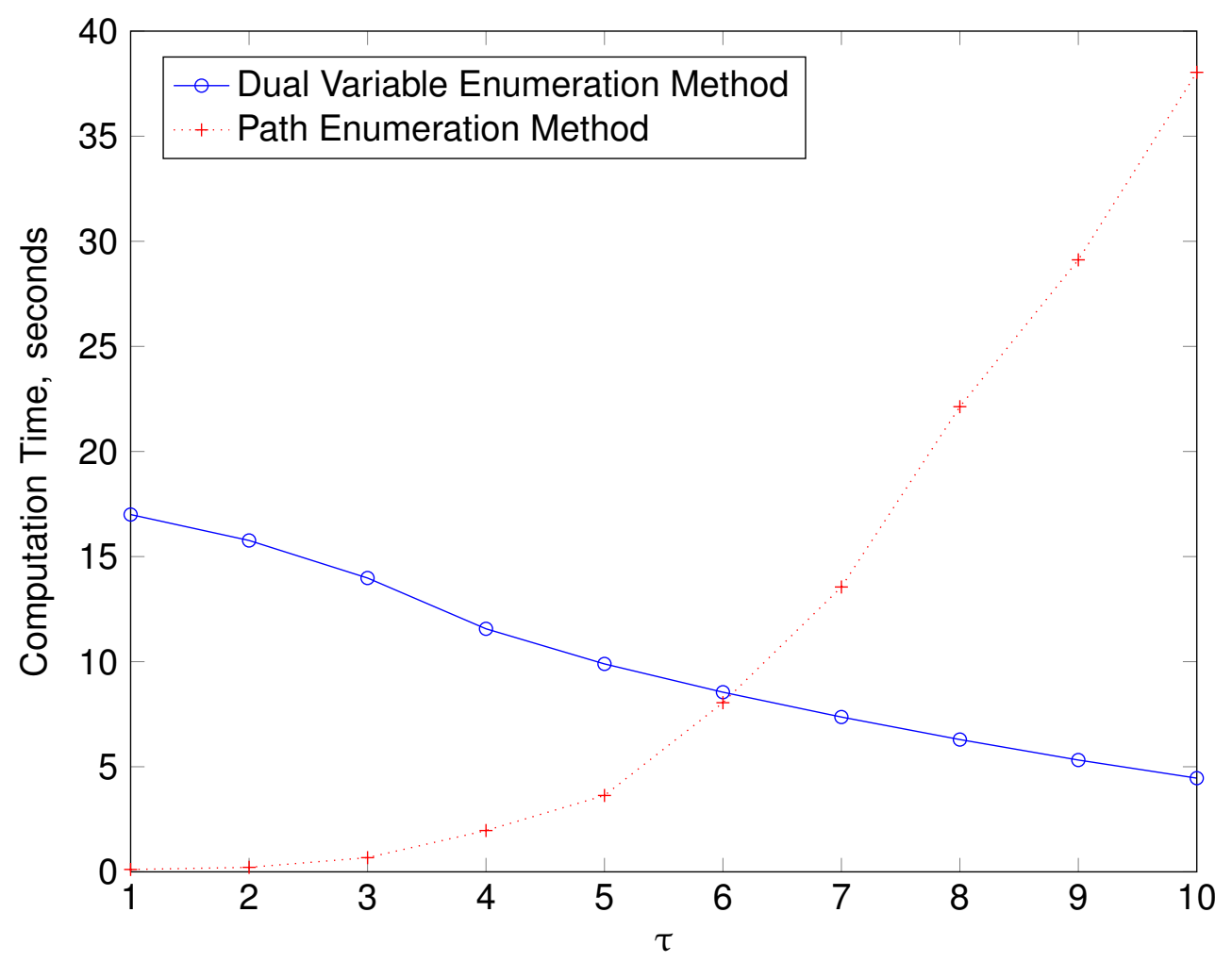

Figure 9: Computation Time for the Two Solution Methods 
useful when the network size and the level of uncertainty are small. While we found an application in hazardous materials transportation, we may apply our results to any risk mitigation problems involving network-structured decision processes.

\section{Acknowledgements}

The first author's research was partially supported by NSF grant CMMI-1068585. The second author's research was supported by research funds of Chonbuk National University in 2011.

\section{References}

[1] M. Abkowitz and PD Cheng. Developing a risk/cost framework for routing truck movements of hazardous materials. Accident Analysis and Prevention, 20(1):39, 1988.

[2] Ravindra K. Ahuja, Kurt Mehlhorn, James Orlin, and Robert E. Tarjan. Faster algorithms for the shortest path problem. J. ACM, 37:213-223, April 1990. ISSN 0004-5411. doi: http://doi.acm.org/10.1145/77600.77615. URL http://doi.acm.org/10.1145/77600.77615.

[3] V. Akgun, A. Parekh, R. Batta, and C. Rump. Routing of a hazmat truck in the presence of weather systems. Computers 85 Operations Research, 34(5): 1351-1373, May 2007. ISSN 03050548. doi: 10.1016/j.cor.2005.06.005. URL http://dx.doi.org/10.1016/j.cor.2005.06.005.

[4] E. Alp. Risk-based transportation planning practice: Overall methodology and a case example. INFOR, 33(1):4-19, 1995.

[5] R. Batta and S.S. Chiu. Optimal obnoxious paths on a network: Transportation of hazardous materials. Operations Research, 36(1):84-92, 1988.

[6] D. Bertsimas and M. Sim. Robust Discrete Optimization Under Ellipsoidal Uncertainty Sets. 2004.

[7] Dimitris Bertsimas and Melvyn Sim. Robust discrete optimization and network flows. Mathematical Programming, 98(1):49-71, September 2003. ISSN 0025-5610. doi: 10.1007/s10107003-0396-4. URL http://dx.doi.org/10.1007/s10107-003-0396-4.

[8] Dimitris Bertsimas and Robert Weismantel. Optimization over integers, volume 13. Dynamic Ideas Belmont, 2005.

[9] D. Chaerani, C. Roos, and A. Aman. The Robust Shortest Path Problem by Means of Robust Linear Optimization. In Hein Fleuren, Dick Hertog, and Peter Kort, editors, Operations Research Proceedings 2004, volume 2004 of Operations Research Proceedings, chapter 42, pages 
335-342-342. Springer Berlin Heidelberg, Berlin/Heidelberg, 2005. ISBN 3-540-24274-0. doi: 10.1007/3-540-27679-3_42. URL http://dx.doi.org/10.1007/3-540-27679-3_42.

[10] C. A. Floudas and V. Visweswaran. Quadratic Optimization, pages 217-270. Kluwer Academic Publishers, 1994.

[11] Alain Ghouila-Houri. Caractérisation des matrices totalement unimodulaires. CR Acad. Sci. Paris, 254:1192-1194, 1962.

[12] H. Jin and R. Batta. Objectives derived from viewing hazmat shipments as a sequence of independent Bernoulli trials. Transportation Science, 31(3):252-261, 1997.

[13] B.Y. Kara and V. Verter. Designing a road network for hazardous materials transportation. Transportation Science, 38(2):188, 2004.

[14] Panos Kouvelis and Gang Yu. Robust Discrete Optimization and Its Applications (Nonconvex Optimization and Its Applications). Springer, 1st edition, November 1996. ISBN 9780792342915. URL http://www.worldcat.org/isbn/0792342917.

[15] D. Lord, A. Manar, and A. Vizioli. Modeling crash-flow-density and crash-flow-v/c ratio relationships for rural and urban freeway segments. Accident Analysis 8 Prevention, 37(1): 185-199, 2005.

[16] J.L. Martin. Relationship between crash rate and hourly traffic flow on interurban motorways. Accident Analysis \& Prevention, 34(5):619-629, 2002.

[17] Luis N. Vicente, Paul H. Calamai, and Joaquim J. Júdice. Generation of disjointly constrained bilinear programming test problems. Computational Optimization and Applications, 1(3):299-306, December 1992. ISSN 0926-6003. doi: 10.1007/BF00249639. URL http://dx.doi.org/10.1007/BF00249639.

[18] J.Y. Yen. Finding the $K$ shortest loopless paths in a network. Management Science, 17(11): 712-716, 1971. ISSN 0025-1909. 